\title{
Three-dimensional relativistic simulations of rotating neutron-star collapse to a Kerr black hole
}

\author{
Luca Baiotti, ${ }^{1}$ Ian Hawke, ${ }^{2}$ Pedro J. Montero, ${ }^{1}$ Frank Löffler, ${ }^{2}$ \\ Luciano Rezzolla, ${ }^{1,3}$ Nikolaos Stergioulas, ${ }^{4}$ José A. Font, ${ }^{5}$ and Ed Seidel ${ }^{6,3,2}$ \\ ${ }^{I}$ SISSA, International School for Advanced Studies and INFN, Via Beirut 2, 34014 Trieste, Italy \\ ${ }^{2}$ Max-Planck-Institut für Gravitationsphysik, Albert-Einstein-Institut, 14476 Golm, Germany \\ ${ }^{3}$ Department of Physics, Louisiana State University, Baton Rouge, LA 70803 USA \\ ${ }^{4}$ Department of Physics, Aristotle University of Thessaloniki, Thessaloniki 54124, Greece \\ ${ }^{5}$ Departamento de Astronomía y Astrofísica, Universidad de Valencia, Dr. Moliner 50, 46100 Burjassot, Spain \\ ${ }^{6}$ Center for Computation and Technology, Louisiana State University, Baton Rouge, LA 70803 USA
}

(Dated: January 30, 2005)

\begin{abstract}
We present a new three-dimensional fully general-relativistic hydrodynamics code using high-resolution shock-capturing techniques and a conformal traceless formulation of the Einstein equations. Besides presenting a thorough set of tests which the code passes with very high accuracy, we discuss its application to the study of the gravitational collapse of uniformly rotating neutron stars to Kerr black holes. The initial stellar models are modelled as relativistic polytropes which are either secularly or dynamically unstable and with angular velocities which range from slow rotation to the mass-shedding limit. We investigate the gravitational collapse by carefully studying not only the dynamics of the matter, but also that of the trapped surfaces, i.e. of both the apparent and event horizons formed during the collapse. The use of these surfaces, together with the dynamical horizon framework, allows for a precise measurement of the black-hole mass and spin. The ability to successfully perform these simulations for sufficiently long times relies on excising a region of the computational domain which includes the singularity and is within the apparent horizon. The dynamics of the collapsing matter is strongly influenced by the initial amount of angular momentum in the progenitor star and, for initial models with sufficiently high angular velocities, the collapse can lead to the formation of an unstable disc in differential rotation. All of the simulations performed with uniformly rotating initial data and a polytropic or ideal-fluid equation of state show no evidence of shocks or of the presence of matter on stable orbits outside the black hole.

PACS numbers: 04.25.Dm, 04.40.Dg, 04.70.Bw, 95.30.Lz, 97.60.Jd
\end{abstract}

\section{INTRODUCTION}

The numerical investigation of gravitational collapse of rotating stellar configurations leading to black-hole formation is a long standing problem in numerical relativity. However, it is through numerical simulations in general relativity that one can hope to improve our knowledge of fundamental aspects of Einstein's theory such as the cosmic censorship hypothesis and black-hole no-hair theorems, along with that of current open issues in relativistic astrophysics research, such as the mechanism responsible for gamma-ray bursts. Furthermore, numerical simulations of stellar gravitational collapse to black holes provide a unique mean of computing the gravitational waveforms emitted in such events, believed to be among the most important sources of detectable gravitational radiation.

However, the modelling of black-hole spacetimes with collapsing matter-sources in multidimensions is one of the most formidable efforts of numerical relativity. This is due, on one hand, to the inherent difficulties and complexities of the system of equations which is to be integrated, the Einstein field equations coupled to the general-relativistic hydrodynamics equations, and, on the other hand, to the immense computational resources needed to integrate the equations in the case of three-dimensional (3D) evolutions. In addition to the practical difficulties encountered in the accurate treatment of the hydrodynamics involved in the gravitational collapse of a rotating neutron star to a black hole, the precise numerical computation of the gravitational radiation emitted in the process is particularly challenging as the energy released in gravitational waves is much smaller than the total rest-mass energy of the system.

The ability to perform long-term numerical simulations of self-gravitating systems in general relativity strongly depends on the formulation adopted for the Einstein equations. The covariant nature of these equations (the "many-fingered time" of relativity) leads to difficulties in constructing an appropriate coordinate representation which would allow for stable and accurate simulations. Over the years, the standard approach has been mainly based upon the unconstrained solution of the $3+1$ ADM formulation of the field equations, which, despite large-scale and dedicated collaborations [1, 2, 3] has gradually been shown to lack the stability properties necessary for longterm numerical simulations. In recent years, however, a considerable effort has been invested in extending the set of ADM equations solved by including at some level the solution of the constraint equations on each spatial hypersurface [4, 5, 6], or by reformulating the ADM approach in order to achieve longterm stability (see, e.g., [7] and references therein). Building on the experience developed with lower-dimensional formulations, Nakamura, Oohara and Kojima [8] presented in 1987 a conformal traceless reformulation of the ADM system which subsequent authors (see, e.g., [9, 10, 11, 12, 13, 14, 15, 16]) gradually showed to be robust enough to accomplish such a goal for different classes of spacetimes, including black holes and neutron stars (both isolated and in coalescing binary systems). The most widespread version developed from this formalism, which we refer to here as the NOK formulation, was given by [9, 10] and is commonly referred to as the BSSN 
formulation.

In addition to the improvements achieved in the formulation of the field equations, successful long-term 3D evolutions of black holes in vacuum have been obtained in the last few years using excision techniques (see, e.g., [17, 18, 19, 20, 21, 22, 23, 24, 25, 26]), although the original idea is much older [27]. In this approach, the spacetime region within the black-hole horizon, and so causally disconnected, can be safely ignored without affecting the evolution outside the horizon as long as suitable boundary conditions are specified at the excision surface. The simulations presented here show the applicability of excision techniques also in non-vacuum spacetimes, namely during the collapse of rotating neutron stars to Kerr black holes. The excision technique, which is applied once the black-hole apparent horizon is found, permits to extend considerably the lifetime of the simulations, at least with the resolutions used here. This, in turn, allows for an accurate investigation of the dynamics of the trapped surfaces formed during the collapse, from which important information on the mass and spin of the black hole, as well as on the amount of energy which is lost to gravitational radiation, can be extracted. While our study was nearing completion, we have learnt that a similar approach has also been implemented with success [28].

The presence of rotation in the collapsing stellar models requires multidimensional investigations, either in axisymmetry or in full 3D. The numerical investigations of blackhole formation (beyond spherical symmetry) started in the early 1980's with the pioneering work of Nakamura [29]. He adopted the $(2+1)+1$ formulation of the Einstein equations in cylindrical coordinates and introduced regularity conditions to avoid divergences at coordinate singularities. Nakamura used a "hypergeometric" slicing condition which prevents the grid points from reaching the singularity when a black hole forms. The simulations could track the evolution of the collapse of a $10 M_{\odot}$ "core" of a massive star with different amounts of rotational energy, up to the formation of a rotating black hole. However, the numerical scheme employed was not accurate enough to compute the emitted gravitational radiation. In subsequent works, Nakamura [30] (see also [8]) considered a configuration consisting of a neutron star with an accreting envelope, which was thought to mimic mass fall-back in a supernova explosion. Rotation and infall velocity were added to such a configuration, simulating an evolution dependent on the prescribed rotation rates and rotation laws.

Later on, in a series of papers [31, 32, 33, 34], Bardeen, Stark and Piran studied the collapse of rotating relativistic polytropes to black holes, succeeding in computing the associated gravitational radiation. The gravitational field and hydrodynamics equations were formulated using the $3+1$ formalism in two spatial dimensions, using the radial gauge and a mixture of singularity-avoiding polar and maximal slicings. The initial model was a spherically symmetric relativistic polytrope of mass $M$ in equilibrium. The gravitational collapse was induced by lowering the pressure in the initial model by a prescribed (and often very large) fraction and by simultaneously adding an angular momentum distribution approximating rigid-body rotation. Their simulations showed that Kerr black-hole formation occurs only for angular mo- menta less than a critical value. Furthermore, the energy $\Delta E$ carried away through gravitational waves from the collapse to a Kerr black hole was found to be $\Delta E / M c^{2}<7 \times 10^{-4}$, the shape of the waveforms being nearly independent of the details of the collapse.

The axisymmetric codes employed in the aforementioned works adopted curvilinear coordinate systems which may lead to long-term numerical instabilities at coordinate singularities. These coordinate problems have deterred researchers from building 3D codes in spherical coordinates. Recently, a general-purpose method (called "cartoon"), has been proposed to enforce axisymmetry in numerical codes based on Cartesian coordinates and which does not suffer from stability problems at coordinate singularities [35]. It should be noted, however, that the stability properties of the cartoon method are not fully understood yet, as discussed by [36]. Using this method, Shibata [37] investigated the effects of rotation on the criterion for prompt adiabatic collapse of rigidly and differentially rotating polytropes to a black hole, finding that the criterion for black-hole formation depends strongly on the amount of angular momentum, but only weakly on its (initial) distribution. The effects of shock heating when using a nonisentropic equation of state (EOS hereafter) are important in preventing prompt collapse to black holes in the case of large rotation rates.

More recently, Shibata [12, 38] has performed axisymmetric simulations of the collapse of rotating supramassive neutron stars to black holes for a wide range of polytropic EOSs and with an improved implementation of the hydrodynamics solver (based on approximate Riemann solvers) with respect to the original implementation used in [37]. Parameterizing the "stiffness" of the EOS through the polytropic index $N$, the final state of the collapse is a Kerr black hole without any noticeable disc formation, when the polytropic index $N$ is in the range $2 / 3 \leq N \leq 2$. Based on the specific angular momentum distribution in the initial star, Shibata has estimated an upper limit to the mass of a possible disc as being less than $10^{-3}$ of the initial stellar mass [38]. Unfortunately, such small masses cannot currently be confirmed with the presently-available resolutions in 3D simulations on uniform grids.

Three-dimensional, fully relativistic simulations of the collapse of supramassive uniformly rotating neutron stars to rotating black holes were presented in [11]. The simulations focused on $N=1$ polytropes and showed no evidence of massive disc formation or outflows. These results are in agreement with those obtained in axisymmetry [12, 38] and with the new simulations reported by [28] (both in axisymmetry and in 3D) which show that for a rapidly rotating polytrope with $J / M^{2}<0.9$ ( $J$ being the angular momentum) all the mass falls promptly into the black hole, with no disc being formed. Hence, all existing simulations agree that massive disc formation from the collapse of neutron stars requires differential rotation, at least for a polytropic EOS with $1 \leq N \leq 2$.

Here, we present the results of new, fully $3 \mathrm{D}$ simulations of gravitational collapse of uniformly rotating neutron stars, both secularly and dynamically unstable, which we model as 
relativistic polytropes. The angular velocities of our sample of initial models range from slow rotation to the massshedding limit. For the first time in such 3D simulations, we have detected the event horizon of the forming black hole and showed that it can be used to achieve a more accurate determination of the black-hole mass and spin than it would be otherwise possible using the area of the apparent horizon. We have also considered several other approaches to measure the properties of the newly formed Kerr black hole, including the recently proposed isolated and dynamical-horizon frameworks. A comparison among the different methods has indicated that the dynamical-horizon approach is simple to implement and yields estimates which are accurate and more robust than those of the equivalent methods.

The simulations are performed with a new general relativistic hydrodynamics code, the Whisky code, in which the Einstein and hydrodynamics equations are finite-differenced on a Cartesian grid and solved using state-of-the-art numerical schemes (a first description of the code was given in [39]). The code incorporates the expertise developed over the past few years in the numerical solution of the Einstein equations and of the hydrodynamics equations in a curved spacetime (see [13, 14], but also [40] and references therein) and is the result of a collaboration among several European Institutes [41].

As mentioned before, we have implemented in the Whisky code a robust excision algorithm which warrants the extension of the lifetime of the simulations far beyond the evolution times when the black holes first form. Our calculations are starting from initially axisymmetric stellar models but are performed in full 3D to allow for departures from the initial axial symmetry. Overall, our results show that the dynamics of the collapsing matter is strongly influenced by the initial amount of angular momentum in the progenitor neutron star, which, when sufficiently high, leads to the formation of an unstable flattened object. All of the simulations performed for realistic initial data and a polytropic equation of state show no evidence of shock formation preventing a prompt collapse to a black hole, nor the presence of matter on stable orbits outside the black hole. It should be remarked, however, that both of these conclusions may change if the initial stellar models are rotating differentially.

The use of numerical grids with uniform spacing and the present computational resources have placed the outer boundary of our computational box in regions of the spacetime where the gravitational waves have not yet reached their asymptotic form. As a result, the information on the gravitational waveforms that we extract through perturbative techniques [42, 43] does not provide interesting information besides the obvious change in the stellar quadrupole moment. Work is now in progress to use mesh refinement techniques [44] to move the outer boundary sufficiently far from the source so that important information can be extracted on the gravitational wave emission produced during the collapse. The results of these investigations will be presented in a companion paper [45].

The paper is organized as follows: Section III describes the formulation we adopt for the Einstein and hydrodynam- ics equations, the way they are implemented in the code and a brief discussion of how the excision techniques can be employed within a hydrodynamical treatment making use of high-resolution shock-capturing (HRSC) schemes. To avoid detracting the reader's attention from the physical problem considered here, we have confined most of the technical details concerning the numerical implementation of the hydrodynamical equations to Appendix A Section III is therefore devoted to describing the various properties of the initial stellar models. The following two Sections, IV and V present our results regarding the dynamics of the collapsing stars and of the trapped surfaces, respectively. In both cases we will be considering and comparing the dynamics of slowly and of rapidly rotating stellar models. The paper ends with Section VI which contains a summary of the results obtained and the perspectives for further investigations. Finally, Appendix $B$ is devoted to presenting some of the tests the code passes with very high accuracy.

We here use a spacelike signature $(-,+,+,+)$ and a system of units in which $c=G=M_{\odot}=1$ (unless explicitly shown otherwise for convenience). Greek indices are taken to run from 0 to 3 , Latin indices from 1 to 3 and we adopt the standard convention for the summation over repeated indices.

\section{BASIC EQUATIONS AND THEIR IMPLEMENTATION}

The Whisky code solves the general relativistic hydrodynamics equations on a 3D numerical grid with Cartesian coordinates. The code has been constructed within the framework of the Cactus Computational Toolkit (see [46] for details), developed at the Albert Einstein Institute (Golm) and at the Louisiana State University (Baton Rouge). This public domain code provides high-level facilities such as parallelization, input/output, portability on different platforms and several evolution schemes to solve general systems of partial differential equations. Clearly, special attention is dedicated to the solution of the Einstein equations, whose matter-terms in non-vacuum spacetimes are handled by the Whisky code. While the Whisky code is entirely new, its initial development has benefitted in part from the release of a public version of the general relativistic hydrodynamics code described in [14, 47], and developed mostly by the group at the Washington University (St. Louis).

The Whisky code, however, incorporates important recent developments regarding, in particular, new numerical methods for the solution of the hydrodynamics equations that have been described in detail in [39] and will be briefly reviewed in Appendix A These include: (i) the Piecewise Parabolic Method (PPM) [48] and the Essentially NonOscillatory (ENO) methods [49] for the cell-reconstruction procedure; (ii) the Harten-Lax-van Leer-Einfeldt (HLLE) [50] approximate Riemann solver, the Marquina flux formula [51]; (iii) the analytic expression for the left eigenvectors [52] and the compact flux formulae [53] for a Roe-type Riemann solver and a Marquina flux formula; (iv) the use of a "method of lines" (MoL) approach for the implementation of high-order time evolution schemes; $(v)$ the possibility to couple the gen- 
eral relativistic hydrodynamics equations with a conformally decomposed three-metric. The incorporation of these new numerical techniques in the code has led to a much improved ability to simulate relativistic stars, as shown in Appendix B which is devoted to code tests.

While the Cactus code provides at each time step a solution of the Einstein equations [13]

$$
G_{\mu \nu}=8 \pi T_{\mu \nu}
$$

where $G_{\mu \nu}$ is the Einstein tensor and $T_{\mu \nu}$ is the stress-energy tensor, the Whisky code provides the time evolution of the hydrodynamics equations, expressed through the conservation equations for the stress-energy tensor $T^{\mu \nu}$ and for the matter current density $J^{\mu}$

$$
\nabla_{\mu} T^{\mu \nu}=0, \quad \nabla_{\mu} J^{\mu}=0 .
$$

In what follows we briefly discuss how both the right and the left-hand side of equations 2.1 are computed within the coupled Cactus/Whisky codes.

\section{A. Evolution of the field equations}

We here give only a brief overview of the system of equations for the evolution of the field equations, but refer the reader to [13] for more details. Many different formulations of the equations have been proposed throughout the years, starting with the ADM formulation in 1962 [54]. As mentioned in the Introduction, we use the NOK [8] formulation, which is based on the ADM construction and has been further developed in [9].

In the ADM formulation [54], the spacetime is foliated with a set of non-intersecting spacelike hypersurfaces. Two kinematic variables relate the hypersurfaces: the lapse function $\alpha$, which describes the rate of advance of time along a timelike unit vector $n^{\mu}$ normal to a spacelike hypersurface, and the shift three-vector $\beta^{i}$ that relates the coordinates of two spacelike hypersurfaces. In this construction the line element reads

$$
d s^{2}=-\left(\alpha^{2}-\beta_{i} \beta^{i}\right) d t^{2}+2 \beta_{i} d x^{i} d t+\gamma_{i j} d x^{i} d x^{j} .
$$

The original ADM formulation casts the Einstein equations into a first-order (in time) quasi-linear [55] system of equations. The dependent variables are the three-metric $\gamma_{i j}$ and the extrinsic curvature $K_{i j}$, with first-order evolution equations given by

$$
\begin{aligned}
\partial_{t} \gamma_{i j}= & -2 \alpha K_{i j}+\nabla_{i} \beta_{j}+\nabla_{j} \beta_{i} \\
\partial_{t} K_{i j}= & -\nabla_{i} \nabla_{j} \alpha+\alpha\left[R_{i j}+K K_{i j}-2 K_{i m} K_{j}^{m}\right. \\
& \left.-8 \pi\left(S_{i j}-\frac{1}{2} \gamma_{i j} S\right)-4 \pi \rho_{\mathrm{ADM}} \gamma_{i j}\right] \\
& +\beta^{m} \nabla_{m} K_{i j}+K_{i m} \nabla_{j} \beta^{m}+K_{m j} \nabla_{i} \beta^{m}
\end{aligned}
$$

Here, $\nabla_{i}$ denotes the covariant derivative with respect to the three-metric $\gamma_{i j}, R_{i j}$ is the Ricci curvature of the three-metric, $K \equiv \gamma^{i j} K_{i j}$ is the trace of the extrinsic curvature, $S_{i j}$ is the projection of the stress-energy tensor onto the spacelike hypersurfaces and $S \equiv \gamma^{i j} S_{i j}$ (for a more detailed discussion, see [56]). In addition to the evolution equations, the Einstein equations also provide four constraint equations to be satisfied on each spacelike hypersurface. These are the Hamiltonian constraint equation

$$
{ }^{(3)} R+K^{2}-K_{i j} K^{i j}-16 \pi \rho_{\mathrm{ADM}}=0,
$$

and the momentum constraint equations

$$
\nabla_{j} K^{i j}-\gamma^{i j} \nabla_{j} K-8 \pi j^{i}=0
$$

In equations 2.4-2.7), $\rho_{\mathrm{ADM}}$ and $j^{i}$ are the energy density and the momentum density as measured by an observer moving orthogonally to the spacelike hypersurfaces.

Details of our particular implementation of the conformal traceless reformulation of the ADM system as proposed by [8, 9, 10] are extensively described in [13, 57] and will not be repeated here. We only mention, however, that this formulation makes use of a conformal decomposition of the threemetric, $\tilde{\gamma}_{i j}=e^{-4 \phi} \gamma_{i j}$, and the trace-free part of the extrinsic curvature, $A_{i j}=K_{i j}-\gamma_{i j} K / 3$, with the conformal factor $\phi$ chosen to satisfy $e^{4 \phi}=\gamma^{1 / 3}$, where $\gamma$ is the determinant of the spatial three-metric $\gamma_{i j}$. In this formulation, in addition to the evolution equations for the conformal three-metric $\tilde{\gamma}_{i j}$ and the conformal traceless extrinsic curvature $\tilde{A}_{i j}$, there are evolution equations for the conformal factor $\phi$, for the trace of the extrinsic curvature $K$ and for the "conformal connection functions" $\tilde{\Gamma}^{i} \equiv \tilde{\gamma}^{i j}{ }_{, j}$. We note that although the final mixed, first and second-order, evolution system for $\left\{\phi, K, \tilde{\gamma}_{i j}, \tilde{A}_{i j}, \tilde{\Gamma}^{i}\right\}$ is not in any immediate sense hyperbolic, there is evidence showing that the formulation is at least equivalent to a hyperbolic system [58, 59, 60]. In the formulation of [9], the auxiliary variables $\tilde{F}_{i}=-\sum_{j} \tilde{\gamma}_{i j, j}$ were used instead of the $\tilde{\Gamma}^{i}$.

In Refs. [13, 61] the improved properties of this conformal traceless formulation of the Einstein equations were compared to the ADM system. In particular, in [13] a number of strongly gravitating systems were analysed numerically with convergent HRSC methods with total-variation-diminishing (TVD) schemes using the equations described in [47]. These included weak and strong gravitational waves, black holes, boson stars and relativistic stars. The results showed that this treatment leads to numerical evolutions of the various strongly gravitating systems which did not show signs of numerical instabilities for sufficiently long times. However, it was also found that the conformal traceless formulation requires grid resolutions higher than the ones needed in the ADM formulation to achieve the same accuracy, when the foliation is made using the " $K$-driver" approach discussed in [62]. Because in longterm evolutions a small error-growth rate is the most desirable property, we have adopted the conformal traceless formulation as our standard form for the evolution of the field equations. 


\section{Gauge choices}

The code is designed to handle arbitrary shift and lapse conditions, which can be chosen as appropriate for a given spacetime simulation. More information about the possible families of spacetime slicings which have been tested and used with the present code can be found in [13, 22]. Here, we limit ourselves to recalling details about the specific foliations used in the present evolutions. In particular, we have used hyperbolic $K$-driver slicing conditions of the form

$$
\left(\partial_{t}-\beta^{i} \partial_{i}\right) \alpha=-f(\alpha) \alpha^{2}\left(K-K_{0}\right),
$$

with $f(\alpha)>0$ and $K_{0} \equiv K(t=0)$. This is a generalization of many well known slicing conditions. For example, setting $f=1$ we recover the "harmonic" slicing condition, while, by setting $f=q / \alpha$, with $q$ an integer, we recover the generalized " $1+\log$ " slicing condition [63]. In particular, all of the simulations discussed in this paper are done using condition (2.8) with $f=2 / \alpha$. This choice has been made mostly because of its computational efficiency, but we are aware that "gauge pathologies" could develop with the " $1+\log$ " slicings [64, 65].

As for the spatial gauge, we use one of the "Gamma-driver" shift conditions proposed in [22] (see also [57]), that essentially act so as to drive the $\tilde{\Gamma}^{i}$ to be constant. In this respect, the "Gamma-driver" shift conditions are similar to the "Gamma-freezing" condition $\partial_{t} \tilde{\Gamma}^{k}=0$, which, in turn, is closely related to the well-known minimal distortion shift condition [66]. The differences between these two conditions involve the Christoffel symbols and are basically due to the fact that the minimal distortion condition is covariant, while the Gamma-freezing condition is not.

In particular, all of the results reported here have been obtained using the hyperbolic Gamma-driver condition,

$$
\partial_{t}^{2} \beta^{i}=F \partial_{t} \tilde{\Gamma}^{i}-\eta \partial_{t} \beta^{i},
$$

where $F$ and $\eta$ are, in general, positive functions of space and time. For the hyperbolic Gamma-driver conditions it is crucial to add a dissipation term with coefficient $\eta$ to avoid strong oscillations in the shift. Experience has shown that by tuning the value of this dissipation coefficient it is possible to almost freeze the evolution of the system at late times. We typically choose $F=\frac{3}{4}$ and $\eta=3$ and do not vary them in time.

\section{B. Evolution of the hydrodynamics equations}

An important feature of the Whisky code is the implementation of a conservative formulation of the hydrodynamics equations [52, 67, 68], in which the set of equations (2.2) is written in a hyperbolic, first-order and flux-conservative form of the type

$$
\partial_{t} \mathbf{q}+\partial_{i} \mathbf{f}^{(i)}(\mathbf{q})=\mathbf{s}(\mathbf{q}),
$$

where $\mathbf{f}^{(i)}(\mathbf{q})$ and $\mathbf{s}(\mathbf{q})$ are the flux-vectors and source terms, respectively [40]. Note that the right-hand side (the source terms) depends only on the metric, and its first derivatives, and on the stress-energy tensor. Furthermore, while the system (2.10) is not strictly hyperbolic, strong hyperbolicity is recovered in a flat spacetime, where $\mathbf{s}(\mathbf{q})=0$.

As shown by [68], in order to write system (2.2) in the form of system (2.10), the primitive hydrodynamical variables (i.e. the rest-mass density $\rho$ and the pressure $p$ (measured in the rest-frame of the fluid), the fluid three-velocity $v^{i}$ (measured by a local zero-angular momentum observer), the specific internal energy $\epsilon$ and the Lorentz factor $W$ ) are mapped to the so called conserved variables $\mathbf{q} \equiv\left(D, S^{i}, \tau\right)$ via the relations

$$
\begin{aligned}
D & \equiv \sqrt{\gamma} W \rho, \\
S^{i} & \equiv \sqrt{\gamma} \rho h W^{2} v^{i}, \\
\tau & \equiv \sqrt{\gamma}\left(\rho h W^{2}-p\right)-D,
\end{aligned}
$$

where $h \equiv 1+\epsilon+p / \rho$ is the specific enthalpy and $W \equiv\left(1-\gamma_{i j} v^{i} v^{j}\right)^{-1 / 2}$. Note that only five of the seven primitive variables are independent.

In order to close the system of equations for the hydrodynamics an EOS which relates the pressure to the rest-mass density and to the energy density must be specified. The code has been written to use any EOS, but all of the tests so far have been performed using either an (isentropic) polytropic EOS

$$
\begin{aligned}
& p=K \rho^{\Gamma}, \\
& e=\rho+\frac{p}{\Gamma-1},
\end{aligned}
$$

or an "ideal fluid" EOS

$$
p=(\Gamma-1) \rho \epsilon .
$$

Here, $e$ is the energy density in the rest-frame of the fluid, $K$ the polytropic constant (not to be confused with the trace of the extrinsic curvature defined earlier) and $\Gamma$ the adiabatic exponent. In the case of the polytropic EOS 2.12, $\Gamma=1+1 / N$, where $N$ is the polytropic index and the evolution equation for $\tau$ needs not be solved. In the case of the ideal-fluid EOS (2.14), on the other hand, non-isentropic changes can take place in the fluid and the evolution equation for $\tau$ needs to be solved. In addition to the EOSs 2.12 and (2.14), a "hybrid" EOS (suitable for core-collapse simulations), as described in [69, 70], has also been implemented.

Note that polytropic EOSs (2.12) do not allow any transfer of kinetic energy to thermal energy, a process which occurs in physical shocks (shock heating). However, we have verified, by performing simulations with the more general EOS (2.14) on some selected cases, that for the physical systems treated here, shock heating is not important (no shocks form during our simulations). Since in our numerical scheme using e general EOS like 2.14 is more expensive than using a polytropic EOS, the systematic investigations presented here have been obtained using the latter.

Additional details of the formulation we use for the hydrodynamics equations can be found in [40]. We stress that an important feature of this formulation is that it has allowed to extend to a general relativistic context the powerful numerical methods developed in classical hydrodynamics, in particular HRSC schemes based on linearized Riemann solvers 
(see [40]). Such schemes are essential for a correct representation of shocks, whose presence is expected in several astrophysical scenarios. Two important results corroborate this view. The first one, by Lax and Wendroff [71], states that a stable scheme converges to a weak solution of the hydrodynamical equations. The second one, by Hou and LeFloch [72], states that, in general, a non-conservative scheme will converge to the wrong weak solution in the presence of a shock, hence underlining the importance of flux-conservative formulations. For a full introduction to HRSC methods the reader is also referred to [73, 74, 75].

\section{Hydrodynamical excision}

Excision boundaries are usually based on the principle that a region of spacetime that is causally disconnected can be ignored without this affecting the solution in the remaining portion of the spacetime. Although this is true for signals and perturbations travelling at physical speeds, numerical calculations may violate this assumption and disturbances, such as gauge waves, may travel at larger speeds thus leaving the physically disconnected regions.

Note that, in non-vacuum spacetimes, the excision boundaries for the hydrodynamical and the metric fields need not be the same. For the fluid quantities, in fact, all characteristics emanating from an event in spacetime will propagate within the sound-cone at that event, and, for physically realistic EOSs, this sound-cone will always be contained within the light-cone at that event. As a result, if a region of spacetime contains trapped surfaces, both the hydrodynamical and the metric fields are causally disconnected and both can be excised there. On the other hand, there may be situations, e.g., when the bulk flow is locally supersonic but no trapped surfaces are present, in which it is possible (at least in principle) to excise the hydrodynamical fields without having to do the same for the metric fields. We have not used this option here and the hydrodynamical excision implemented in our simulations has always been made within regions of the spacetime contained inside trapped surfaces.

A first naive implementation of an excision algorithm within a HRSC method could ensure that the data used to construct the flux at the excision boundary is extrapolated from data outside the excision region. This may appear to be a good idea since HRSC methods naturally change the stencils depending on the data locally. In general, however, this approach is not guaranteed to reduce the total variation of the solution and simple examples may be produced that fail with this boundary condition.

An effective solution, however, is not much more complicated and can be obtained by applying at the excision boundary the simplest outflow boundary condition (here, by outflow we mean flow into the excision region). In practice, we apply a zeroth-order extrapolation to all variables at the boundary, i.e. a simple copy of the hydrodynamical variables across the excision boundary (note that setting the hydrodynamical fields inside the excised region to zero would still yield an outflow boundary condition, but leads to incorrect outflow speeds). If the reconstruction method requires more cells inside the excision region, we force the stencil to only consider the data in the exterior and the first interior cell. Although the actual implementation of this excision technique may depend on the reconstruction method used, the working principle is always the same.

The location of the excision boundary itself is based on the determination of the apparent horizon which, within the Cactus code, is obtained using the fast finder of Thornburg [76]. The excision boundary is placed a few gridpoints (typically 4), within a surface which is 0.6 times the size of the apparent horizon. This may not be a suitable outflow boundary on a Cartesian grid, as pointed out by [77, 78]. However, similar or larger excision regions show no problems in vacuum evolutions and since the sound-cones are always contained within the light-cones, we expect no additional problems to arise from the hydrodynamics.

More details on how the hydrodynamical excision is applied in practice, as well as tests showing that this method is stable, consistent and converges to the expected order will be published in a separate paper [79].

\section{INITIAL STELLAR MODELS}

As mentioned earlier, this paper is specially dedicated to study the gravitational collapse of slowly and rapidly rotating supramassive relativistic stars, in uniform rotation, that have become unstable to axisymmetric perturbations. Given equilibrium models of gravitational mass $M$ and central energy density $e_{c}$ along a sequence of fixed angular momentum or fixed rest mass, the Friedman, Ipser \& Sorkin criterion $\partial M / \partial e_{c}=0$ [80] can be used to locate the exact onset of the secular instability to axisymmetric collapse. The onset of the dynamical instability to collapse is located near that of the secular instability but at somewhat larger central energy densities. Unfortunately, no simple criterion exists to determine this location, but the expectation mentioned above has been confirmed by the simulations performed here and by those discussed in [11]. Note that in the absence of viscosity or strong magnetic fields, the star is not constrained to rotate uniformly after the onset of the secular instability and could develop differential rotation. In a realistic neutron star, however, viscosity or intense magnetic fields are likely to enforce a uniform rotation and cause the star to collapse soon after it passes the secular instability limit.

The initial data for our simulations are constructed using a 2D numerical code, that computes accurate stationary equilibrium solutions for axisymmetric and rapidly rotating relativistic stars in polar coordinates [81]. The data are then transformed to Cartesian coordinates using standard coordinate transformations. The same initial data routines have been used in previous 3D simulations [13, 14, 82] and details on the accuracy of the code can be found in [83].

For simplicity, we have focused on initial models constructed with the polytropic EOS 2.12), choosing $\Gamma=2$ and polytropic constant $K_{\mathrm{ID}}=100$ to produce stellar models that are, at least qualitatively, representative of what is expected 


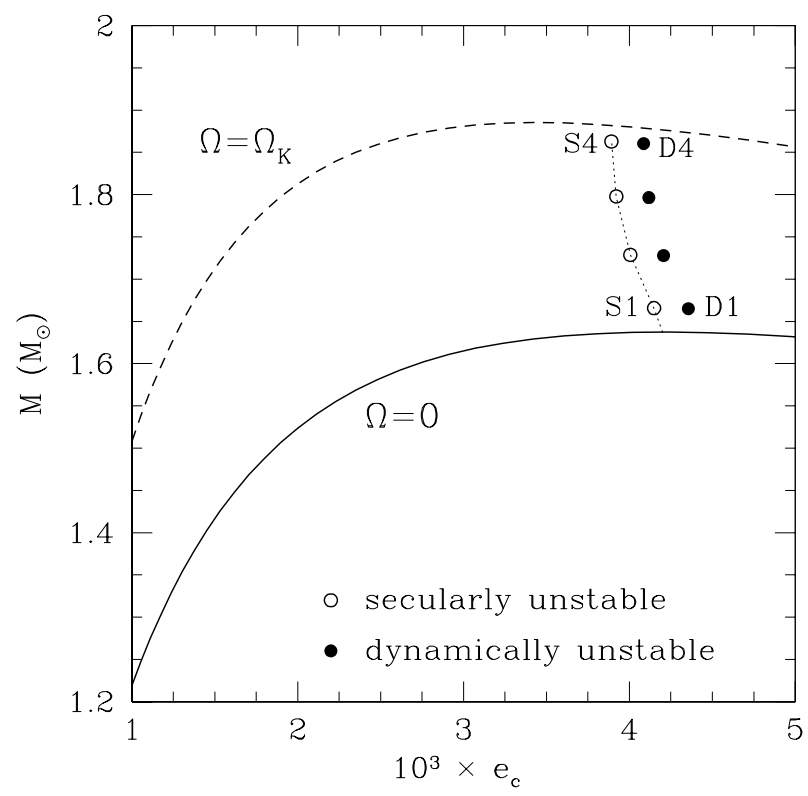

FIG. 1: Gravitational mass shown as a function of the central energy density for equilibrium models constructed with the polytropic EOS, for $\Gamma=2$ and polytropic constant $K_{\mathrm{ID}}=100$. The solid, dashed and dotted lines correspond to the sequence of non-rotating models, the sequence of models rotating at the mass-shedding limit and the sequence of models that are at the onset of the secular instability to axisymmetric perturbations. Also shown are the secularly (open circles) and dynamically unstable (filled circles) initial models used in the collapse simulations.

from observations of neutron stars. More specifically, we have selected four models located on the line defining the onset of the secular instability and having polar-to-equatorial axes ratio of roughly $0.95,0.85,0.75$ and 0.65 (these models are indicated as S1-S4 in Fig 1, respectively. Four additional models were defined by increasing the central energy density of the secularly unstable models by $5 \%$, keeping the same axis ratio. These models (indicated as D1-D4 in Fig 1) were expected and have been found to be dynamically unstable.

Figure 1 shows the gravitational mass as a function of the central energy density for equilibrium models constructed with the chosen polytropic EOS. The solid, dashed and dotted lines correspond respectively to: the sequence of nonrotating models, the sequence of models rotating at the massshedding limit and the sequence of models that are at the onset of the secular instability to axisymmetric perturbations. Furthermore, the secularly and dynamically unstable initial models used in the collapse simulations are shown as open and filled circles, respectively.

Table凹 summarizes the main equilibrium properties of the initial models. The circumferential equatorial radius is denoted as $R_{e}$, while $\Omega$ is the angular velocity with respect to an inertial observer at infinity, and $r_{p} / r_{e}$ is the ratio of the polar to equatorial coordinate radii. The height of the corotating innermost stable circular orbit (ISCO) is defined as $h_{+}=R_{+}-R_{e}$, where $R_{+}$is the circumferential radius
TABLE I: Equilibrium properties of the initial stellar models. The different columns refer respectively to: the central rest-mass density $\rho_{c}$, the ratio of the polar to equatorial coordinate radii $r_{p} / r_{e}$, the gravitational mass $M$, the circumferential equatorial radius $R_{e}$, the angular velocity $\Omega$, the ratio $J / M^{2}$ where $J$ is the angular momentum, the ratio of rotational kinetic energy to gravitational binding energy $T /|W|$, and the "height" of the corotating ISCO $h_{+}$. All models have been computed with a polytropic EOS with $K_{\text {ID }}=100$ and $\Gamma=2$.

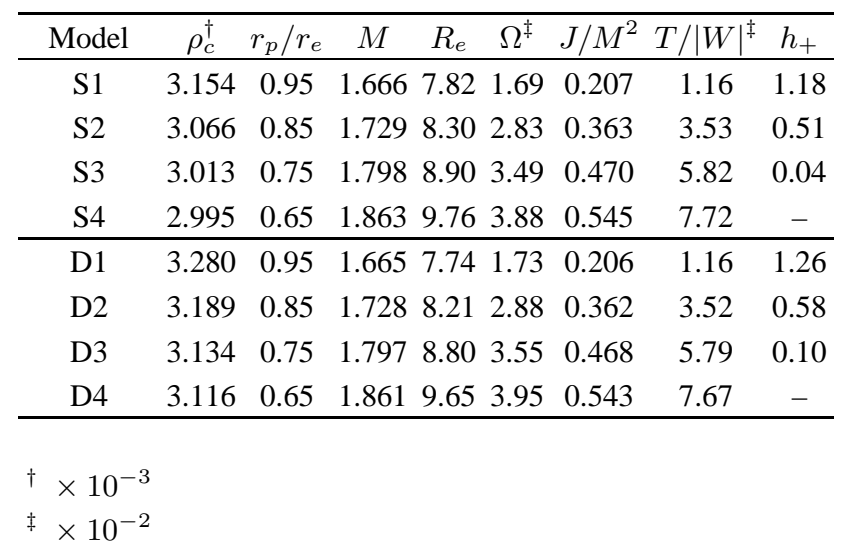

for a corotating ISCO observer. Note that in those models for which a value of $h_{+}$is not reported, all circular geodesic orbits outside the stellar surface are stable. Other quantities shown are the central rest-mass density $\rho_{c}$, the angular momentum $J$, and the ratio of rotational kinetic energy to gravitational binding energy $T /|W|$.

\section{DYNAMICS OF THE MATTER}

This Section discusses the dynamics of the matter during the collapse of the initial stellar models described in the preceding section. All of the simulations reported here have been computed using a uniformly spaced computational grid for which symmetry conditions are imposed across the equatorial plane. Different spatial resolutions have been used to check convergence and improve the accuracy of the results, with the finest resolution having been obtained using $288^{2} \times 144$ cells. While the precise numbers depend on the resolution used and on the model simulated, as a general rule we have used $\sim 50 \%$ of the cells in the $x$-direction to cover the star in case D1 and $\sim 66 \%$ of the cells in the $x$-direction to cover the star in D4. As a result, the outer boundary is set at $\sim 2.0$ times the stellar equatorial radius for D1 and at $\sim 1.4$ times the stellar equatorial radius for D4.

The hydrodynamics equations have been solved employing the Marquina flux formula and a third-order PPM reconstruction, which was shown in [84] to be superior to other methods in maintaining a highly-accurate angular-velocity profile (see also [82, 85] for recent 3D evolutions of rotating relativistic stars with a third-order order PPM reconstruction). The Einstein field equations, on the other hand, have been evolved using the ICN evolution scheme, the " $1+\log$ " slic- 


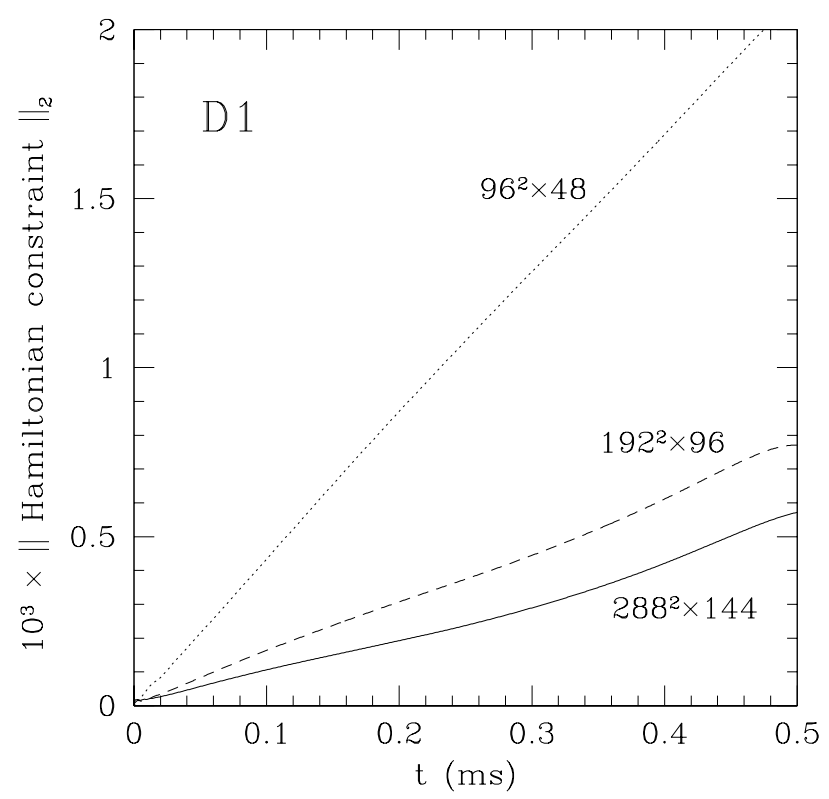

FIG. 2: $L_{2}$ norm of the Hamiltonian constraint violation for the initial model D1 shown as a function of time. The different lines refer to different grid resolutions, but in all cases the IVP was solved after the pressure was uniformly decreased to trigger the collapse.

ing condition and the "Gamma-driver" shift conditions [22]. Finally, both polytropic and ideal-fluid EOSs have been used, although no significant difference has been found in the dynamics between the two cases. This is most probably related to the small $J / M^{2}$ of the uniformly rotating initial models considered here. This implies a relatively rapid collapse and as a result we do not see any shock formation (see below for a more complete discussion). Hereafter we will restrict our attention to a polytropic EOS only.

Given an initial stellar model which is dynamically unstable, simple round-off errors would be sufficient to produce an evolution leading either to the gravitational collapse to a black hole or to the migration to the stable branch of the equilibrium configurations [14] (we recall that both evolutions are equally probable mathematically, although it is easier to imagine that it would collapse to a black hole). In general, however, leaving the onset of the dynamical instability to the cumulative effect of the numerical truncation error is not a good idea, since this produces instability-growth times that are dependent on the grid-resolution used.

For this reason, we induce the collapse by slightly reducing the pressure in the initial configuration. This is done uniformly throughout the star by using a polytropic constant for the evolution $K$ that is smaller than the one used to calculate the initial data $K_{\mathrm{ID}}$. The accuracy of the code is such that only very small perturbations are sufficient to produce the collapse and we have usually adopted $\left(K_{\mathrm{ID}}-K\right) / K_{\mathrm{ID}} \lesssim 2 \%$.

After imposing the pressure reduction, the Hamiltonian and momentum constraints are solved to enforce that the constraint violation is at the truncation-error level. We refer to this procedure as to solving the initial value problem (IVP), which ensures that second order convergence holds from the start of the simulations, as shown in Fig. 2 for the $L_{2}$ norm of the Hamiltonian constraint. Strict second-order convergence is lost when the excision is introduced, although the code remains convergent at a lower rate while the norms of the Hamiltonian constraint start to grow exponentially (this is not shown in Fig. 2]. We are presently investigating the origin of the deterioration of the convergence rate at the time of excision, although this is somewhat unavoidable when excising a spherical region in a Cartesian rectangular grid in the course of the evolution.

Details on how we solve the IVP implementing the YorkLichnerowicz conformal transverse-traceless decomposition can be found in [86]. If, on the other hand, the IVP is not solved after the pressure change, the constraints violation increases twice as fast and converges to second order only after an initial period of about $20 M \sim 0.17 \mathrm{~ms}$. To assess the validity of our procedure to trigger the collapse, we also perform the pressure change after the evolution has started and without solving the IVP. In this case, after the system has recovered from the perturbation, the violation of the constraints is only a few percent different from the case in which the IVP is solved. Furthermore, other dynamical features of the collapse, such as the instant at which the apparent horizon is first formed (see Section $\nabla$ for a detailed discussion), do not vary by more than $1 \%$.

The dynamics resulting from the collapse of models S1S4 and D1-D4 are extremely similar and no qualitative differences have been detected. However, as one would expect, models D1-D4 collapse more rapidly to a black hole (the formation of the apparent horizon appears about 5\% earlier in coordinate time), are computationally less expensive and therefore better suited for a detailed investigation. As a result, in what follows we will restrict our discussion to the collapse of the dynamically unstable models and distinguish the dynamics of case D1, in Section IVA from that of model D4, in SectionIVB

\section{A. Slowly rotating stellar models}

We start by discussing the dynamics of the matter by looking at the evolution of the initial stellar model D1 which is slowly rotating (thus almost spherical, with $r_{p} / r_{e}=0.95$ ) and has the largest central density ( $c f$. Fig. 1)and Table \.

We show in Figs. 3 4 some representative snapshots of the evolution of this initial model. The different panels of Fig. 3. in particular, refer to the initial and intermediate stages of the collapse and show the isocontours of the rest-mass density and velocity field in the $(x, y)$ plane (left column) and in the $(x, z)$ plane (right column), respectively. The isobaric surfaces are logarithmically spaced starting from $\rho=2.0 \times 10^{-5}$ and going up to $\rho=2.0 \times 10^{-3}$ at the stellar interior. The velocity vector field is expressed in units of $c$ and the length for a velocity of $0.2 c$ is shown in the lower-right panel. Panels on the same row refer to the same instant in time and this is indicated in $\mathrm{ms}$ in the top-right corner of each panel. The units on both 

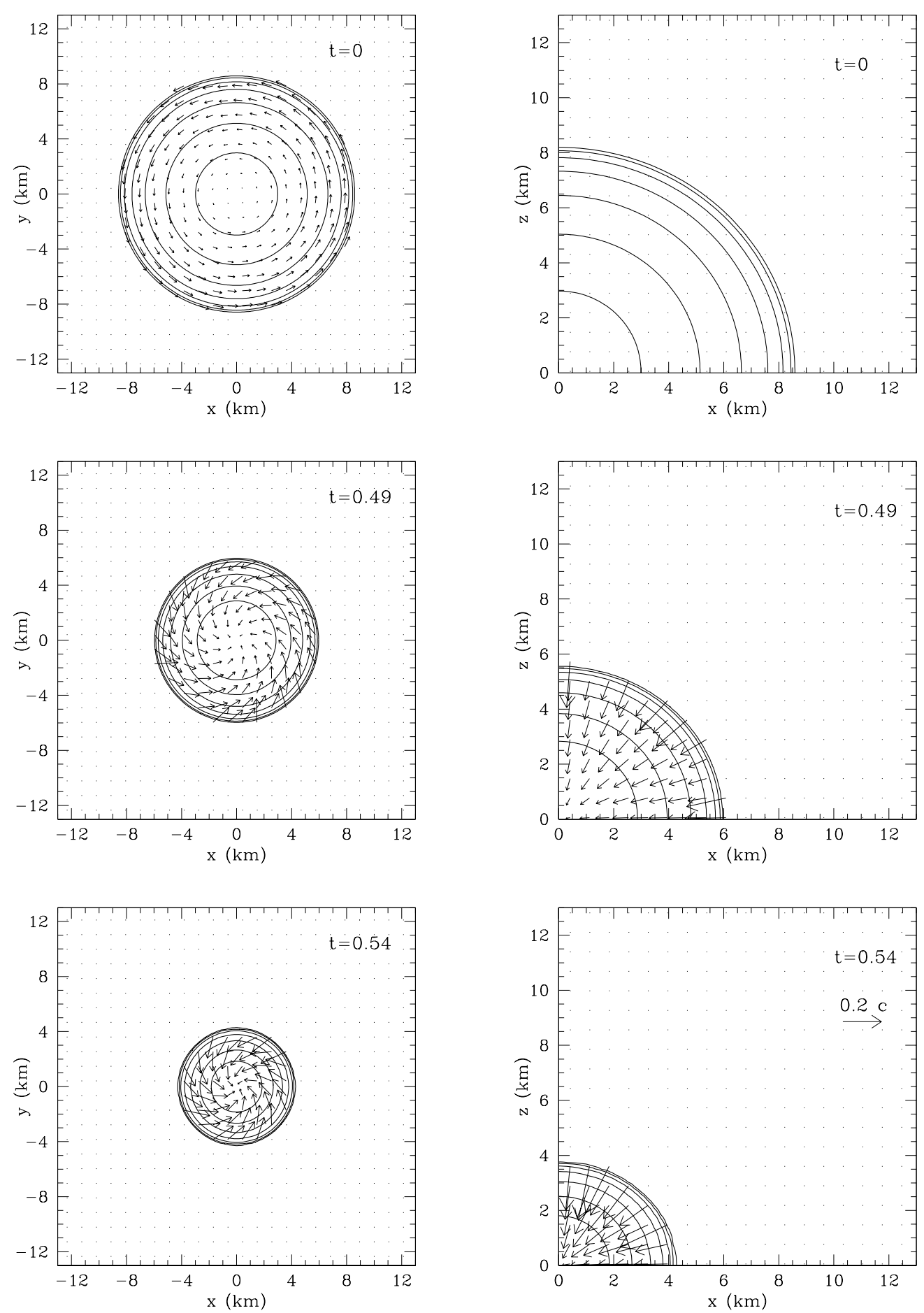

FIG. 3: Collapse sequence for the slowly rotating model D1. Different panels refer to different snapshots during the collapse and show the isocontours of the rest-mass density and velocity field in the $(x, y)$ plane (left column) and in the $(x, z)$ plane (right column), respectively. The isobaric surfaces are logarithmically spaced and a reference length for the vector field is shown in the lower right panel for a velocity of $0.2 \mathrm{c}$. The time of the different snapshots appears in the top right corner of each panel and it is given in ms, while the units on both axes are expressed in km. See main text for a discussion and Fig. 5 for a comparison with the collapse of a rapidly rotating model. 

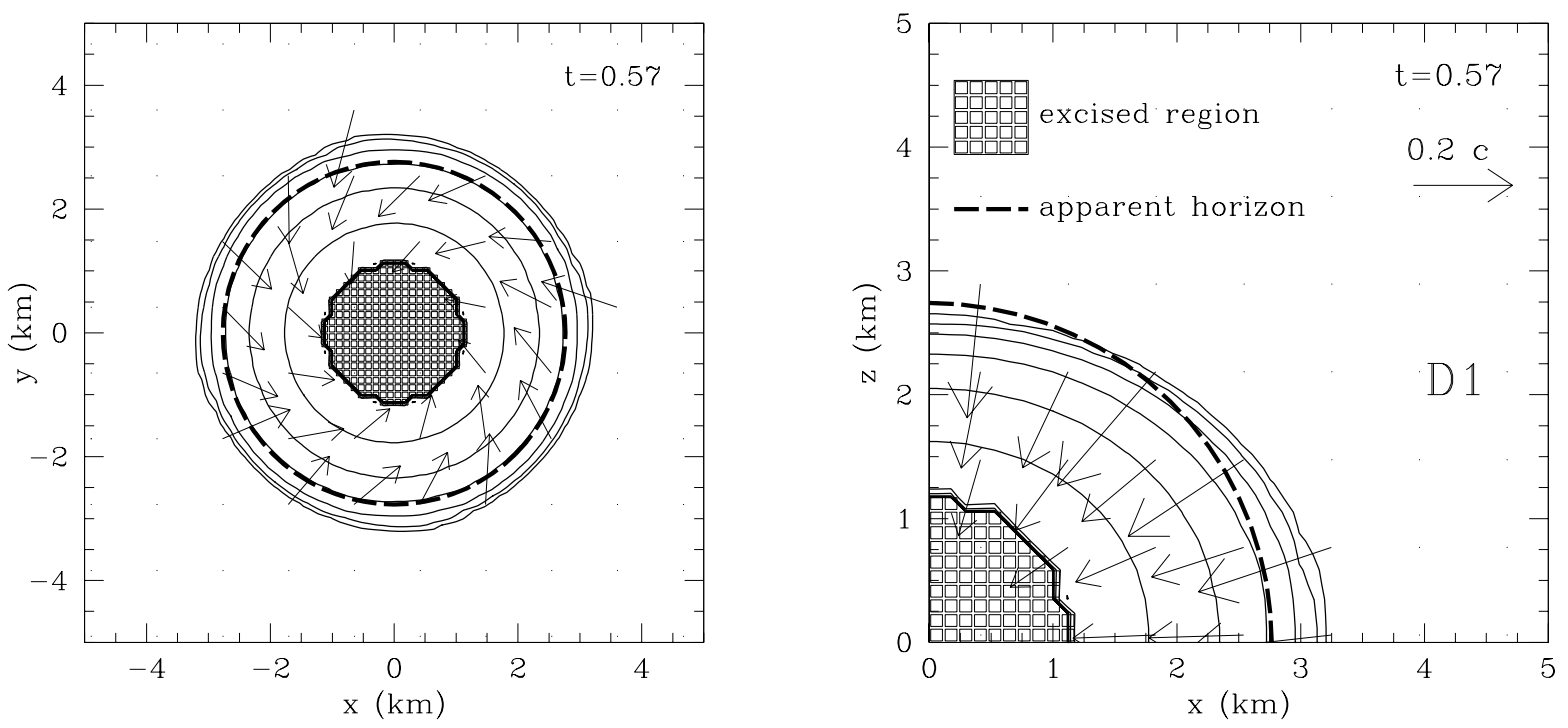

FIG. 4: Magnified view of the final stages of the collapse of model D1. A region around the singularity that has formed is excised from the computational domain and is shown as an area filled with squares. Also shown with a thick dashed line is the coordinate location of the apparent horizon. Note that because of rotation this surface is not a two-sphere, although the departures are not significant and cannot be appreciated from the Figure ( $c f$. Fig. 16 for a clearer view).

axes are expressed in $\mathrm{km}$ and refer to coordinate lengths. This sequence has been obtained with a grid of $288^{2} \times 144$ zones but the data for the velocity field has been down-sampled to produce clearer figures. The data have also been restricted to a single octant in the $(x, z)$ plane to provide a magnified view. In all cases, however, the whole extent of the numerical grid is reported in the figures.

Overall, the sequence shown in Fig. 3is simple to illustrate. During the collapse of model D1 spherical symmetry is almost preserved; as the star increases its compactness and the matter is compressed to larger pressures, the velocity field acquires a radial component (which was zero initially) that grows to relativistic values. This is clearly shown in the panels at $t=0.49$ $\mathrm{ms}$ and $t=0.54 \mathrm{~ms}$, which indicate that the star roughly preserves the ratio of its polar $r_{p}$ and equatorial $r_{e}$ radii (see also Fig. 7, while radial velocities in excess of $\sim 0.28 c$ can be easily reached. The behaviour of the angular velocity during this collapse will be analysed in more detail in Section IVC but we can here anticipate that it does not show appreciable departures from a profile which is uniform inside the star.

Soon after $t=0.54 \mathrm{~ms}$, (i.e. at $t=0.546 \mathrm{~ms}=66.6 M$ in the high-resolution run), an apparent horizon is found and when this has grown to a sufficiently large area, the portion of the computational domain containing the singularity is excised. A discussion of how the trapped surfaces are studied in practice will be presented in Section $\nabla$, while details on the hydrodynamical excision have been given in Section $\amalg \mathrm{C}$ Here, we just remark that the use of an excised region and the removal of the singularity from the computational domain is essential for extending the calculations significantly past this point in time. Figure 4 shows a magnified view of the final stages of the collapse of model D1. Indicated as an area filled with squares is the excised region of the computational do- main, which is an approximation of a sphere on the uniform Cartesian grid, i.e. a "lego-sphere". Also shown with a thick dashed line is the coordinate location of the apparent horizon and it should be remarked that, because of rotation, this surface is not a coordinate two-sphere, although the departures are not significant and cannot be appreciated in Fig. 4 (see Section $\nabla$ and Table $\prod$ for details). At $t=0.57 \mathrm{~ms}$, the time which Fig. 4 refers to, most of the matter has already fallen within the apparent horizon and has assumed an oblate shape.

The numerical calculations were carried out up to $t \simeq 0.73 \mathrm{~ms} \sim 89 \mathrm{M}$, thus using an excised region in a dynamical spacetime for more than $26 \%$ of the total computing time. By this point, all of the stellar matter has collapsed well within the event horizon and the Hamiltonian constraint violation has become very large.

Overall, confirming what was already discussed by several authors in the past, the gravitational collapse of the slowly rotating stellar model D1 takes place in an almost spherical manner and we have found no evidence of shock formation which could prevent the prompt collapse to a black hole, nor appreciable deviations from axisymmetry ( $c f$. left panel of Fig. 44) It is possible, although not likely, that these qualitative features may be altered when a realistic EOS is used, since in this case shocks may appear, whose heating could stall or prevent the prompt collapse to a black hole. However, as mentioned in the Introduction, more dramatic changes are expected to appear if the initial configurations are chosen to have larger initial angular momenta and in particular when $J / M^{2} \gtrsim 1[12,28]$. A first anticipation of the important corrections that centrifugal effects could produce is presented in the following Section, where we examine the dynamics of a rapidly rotating stellar model. 

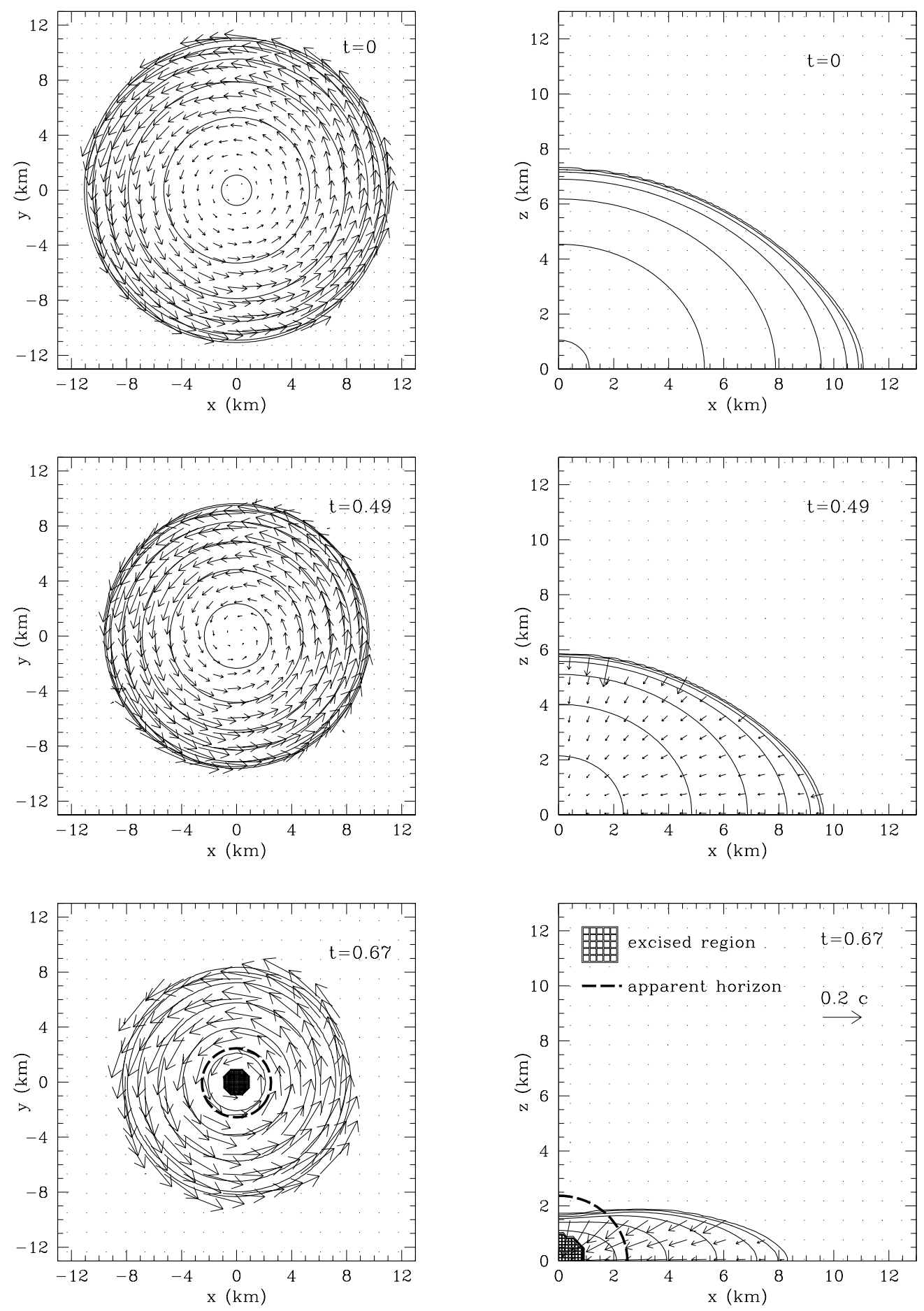

FIG. 5: Collapse sequence for the rapidly rotating model D4. The conventions used in these panels are the same as in Fig. 3 which can be used for a comparison with the collapse of a slowly rotating model. Note that a region around the singularity that has formed is excised from the computational domain and is indicated as an area filled with squares. Also shown with a thick dashed line is the coordinate location of the apparent horizon.

\section{B. Rapidly rotating stellar models}

We next consider the dynamics of the matter during the collapse of model D4 which, being rapidly rotating, is already rather flattened initially (i.e. $r_{p} / r_{e}=0.65$ ) and has the largest $J / M^{2}$ among the dynamically unstable models ( $c f$. Fig. 11 and Table【.

As for the slowly rotating star D1, we show in Figs. 5- 6 

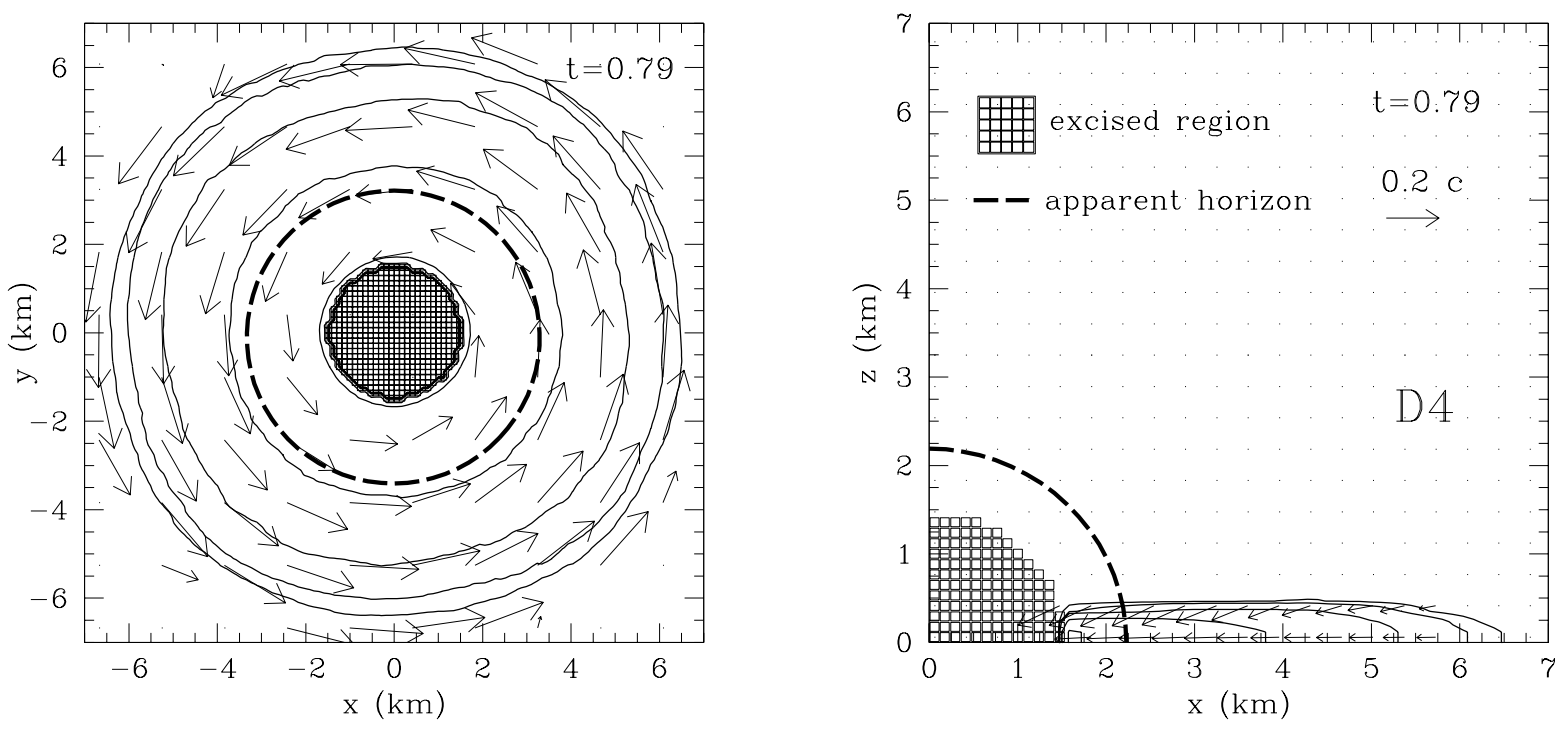

FIG. 6: Magnified view of the final stages of the collapse of model D4. Note that a region around the singularity that has formed is excised from the computational domain and this is indicated as an area filled with squares. Also shown with a thick dashed line is the coordinate location of the apparent horizon. Note that because of the rapid rotation, this surface has significant departures from a two-sphere ( $c f$. Fig. 16 for a clearer view).

some representative snapshots of the evolution of this rapidly rotating model. The data has been computed using the same resolution of $288^{2} \times 144$ zones and the isocontour levels shown for the rest-mass density are the same used in Fig. 3. 4 It is apparent from the panels of Fig. [5 referring to $t=0$, that model D4 is considerably more oblate than D1, as one would expect for a star rotating at almost the mass-shedding limit.

Also in this case, we believe that the sequence in Fig. 5 is simple to illustrate. Note, in particular, how the dynamics is very similar to the one discussed for model D1 up to a time $t \sim 0.49 \mathrm{~ms}$. However, as the collapse proceeds, significant differences between the two models start to emerge and in the case of model D4 the large angular velocity of the progenitor stellar model produces significant deviations from a spherical infall. Indeed, the parts of the star around the rotation axis that are experiencing smaller centrifugal forces collapse more promptly and, as a result, the configuration increases its oblateness.

This is illustrated in Fig. [7, which shows the time evolution of the ratio of the polar and equatorial proper radii for all models in Table \(note that these ratios should not be confused with those in Table \ that refer, instead, to coordinate radii). Each curve in Fig. (7) extends until all of the matter along the $z$-axis has fallen inside the apparent horizon of the newly formed black hole. Clearly, in all cases the oblateness increases as the collapse proceeds and this is much more evident for those stellar models that are rapidly rotating initially. In particular, for the most rapidly rotating models D4 and S4, the ratio between polar and equatorial proper radii becomes as small as 0.45 at the time when the matter on the rotation axis is below the apparent horizon.

At about $t=0.64 \mathrm{~ms}$ (i.e. at $t=0.649 \mathrm{~ms}=70.8 \mathrm{M}$ in the high-resolution run), the collapse of model D4 produces

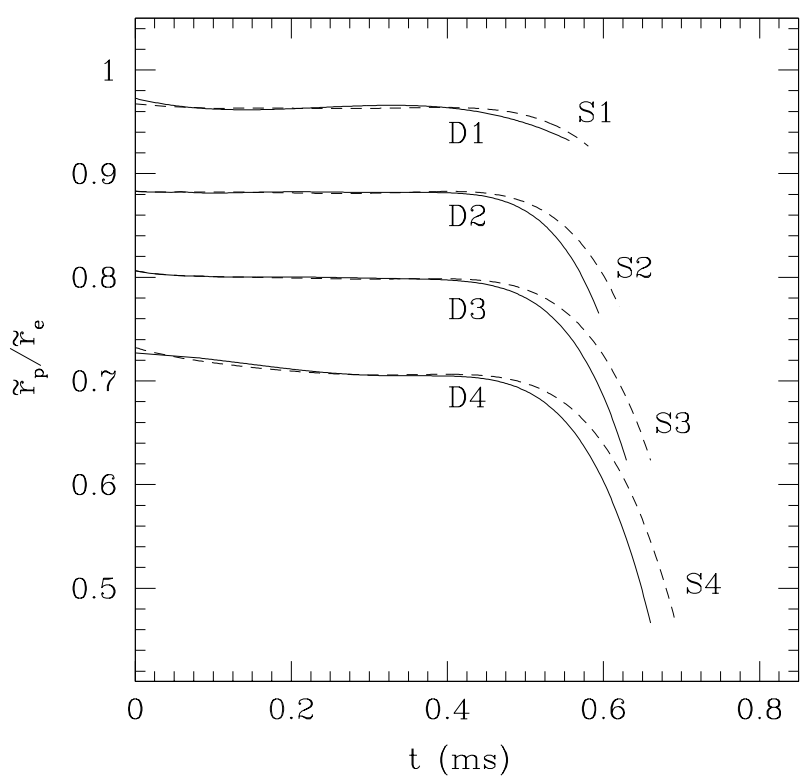

FIG. 7: Ratio of the proper polar radius to the proper equatorial radius for all the initial models. Each curve ends at the time when, for each simulation, all the matter along the $z$-axis has fallen below the apparent horizon.

an apparent horizon. Soon after this, the central regions of the computational domain are excised, preventing the code from crashing and thus allowing for an extended time evolution. The dynamics of the matter at this stage is shown in the lower panels of Fig. [5 which refer to $t=0.67 \mathrm{~ms}$ and where both 
the location of the apparent horizon (thick dashed line) and of the effective excised region (area filled with squares) are shown. By this time the star has flattened considerably, all of the matter near the rotation axis has fallen inside the apparent horizon, but a disc of low-density matter remains near the equatorial plane, orbiting at very high velocities $\gtrsim 0.2 c$. The appearance of an effective barrier preventing a purely radial infall of matter far from the rotational axis may be the consequence of the larger initial angular momentum of the this collapsing matter and of the pressure wave originating from the faster collapse along the ritational axis. Note, in fact, that the radial velocity at the equator does not increase significantly at the stellar surface between $t \simeq 0.49$ and $t \simeq 0.67 \mathrm{~ms}$, but that it actually slightly decreases $(c f$. the $(x, z)$ planes in the mid and lower panels of Fig. 5. This is the opposite of what happens for the radial velocity of the fluid elements in the stellar interior on the equatorial plane: it grows also in this time interval. A more detailed discussion of this behaviour will be made in Section VD

Note that the disc formed outside the apparent horizon is not dynamically stable and, in fact, it rapidly accretes onto the newly formed black hole. This is shown in Fig. 6 which offers a magnified view at a later time $t=0.79 \mathrm{~ms}$. At this stage the disc is considerably flattened but also has large radial inward velocities which induce it to be accreted rapidly onto the black hole. Note that as the area of the apparent horizon increases, so does the excised region, which is allowed to grow accordingly. This can be appreciated by comparing the areas filled with squares in the lower panels of Fig. 5 (referring to $t=0.67 \mathrm{~ms}$ ) with the corresponding ones in Fig. 6 (referring to $t=0.79 \mathrm{~ms}$ ).

By a time $t=0.85 \mathrm{~ms}$, essentially all (i.e. more than $99.9 \%$ ) of the residual stellar matter has fallen within the trapped surface of the apparent horizon and the black hole thus formed approaches the Kerr solution (see Section $\mathrm{V}$. Note that a simple kinematic explanation can be given for the instability of the disc formed during this oblate collapse and comes from relating the position of the outer edge of the disc when this first forms, with the location of the ISCO of the newly formed Kerr black hole. Measuring accurately the mass and spin of the black hole reveals, in fact, that the ISCO is located at $x=11.08 \mathrm{~km}$, which is always larger than the outer edge of the disc ( $c f$. lower panels of Fig. 5). Such behaviour is not surprising since we are here dealing with initial models with a moderate $J / M^{2}$, that collapse essentially on a dynamical timescale, and for which pressure gradients cannot play an important role. As a result, simple point-like particle motion in stationary spacetimes is a sufficient approximation to the dynamics.

Also for model D4, a more detailed discussion (e.g. of the evolution of the distribution of angular velocity, or of the disc rest mass) will be presented in Section IVC Here, however, we can anticipate that when analysed more closely the rest mass in the disc and outside the apparent horizon is effectively very small and that the angular velocity shows appreciable departures from a uniform profile.

A more quantitative description of the rest-mass density evolution is presented in Fig. 8, where different lines show

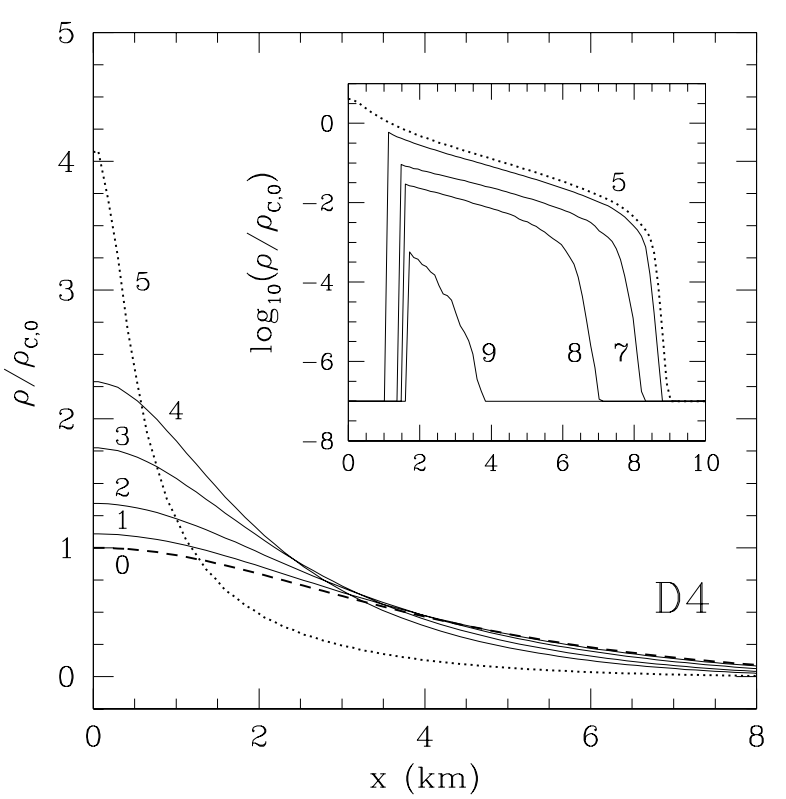

FIG. 8: Rest-mass density of model D4 normalized to the initial value at the stellar centre. The profiles are measured along the $x$-axis on the equatorial plane and refer to different times (see main text for details). Line 5, shown as dotted, corresponds to the time when the apparent horizon is first found. The inset shows a magnified view of the final stages of the evolution using a logarithmic scale and also the location of the excised region as it grows in time.

the profiles of the rest-mass density along the $x$-axis on the equatorial plane. The values are normalized to the initial value at the stellar centre, with different labels referring to different times and in particular to $t=0.0$ (dashed line), $0.25,0.40,0.49,0.54,0.65,0.67,0.74,0.79$ and $0.89 \mathrm{~ms}$, respectively. Line 5, furthermore, is shown as dotted and refers to the time when the apparent horizon is first formed. After this time, the excised region is cut from the computational domain as shown in the inset of Fig. 8 , which illustrates the final stages of the evolution. Note that as the matter falls into the black hole, the apparent horizon increases its radius and thus the location of the excised region moves outside. This is clearly shown in the inset. Note also that the rest-mass density does not drop to zero outside the stellar matter but is levelled off to the uniform value of the atmosphere, whose rest-mass density is seven orders of magnitude smaller than the initial central density.

It should be remarked that such a tenuous atmosphere has no dynamical impact and does not produce any increase of the mass of the black hole that can be appreciated in our simulations. With such rest-mass densities, in fact, it would take a time $\sim 10^{4} M$ to produce a net increase of $\sim 1 \%$ in the blackhole mass. Clearly, these systematic errors are well below the truncation errors, even at the highest resolutions.

The simulation ends at $t=0.91 \mathrm{~ms} \sim 99 \mathrm{M}$, when the rest-mass density is everywhere at the atmosphere level and the violations of the Hamiltonian constraint are large. By this time the evolution has been carried for more than $28 \%$ of the 

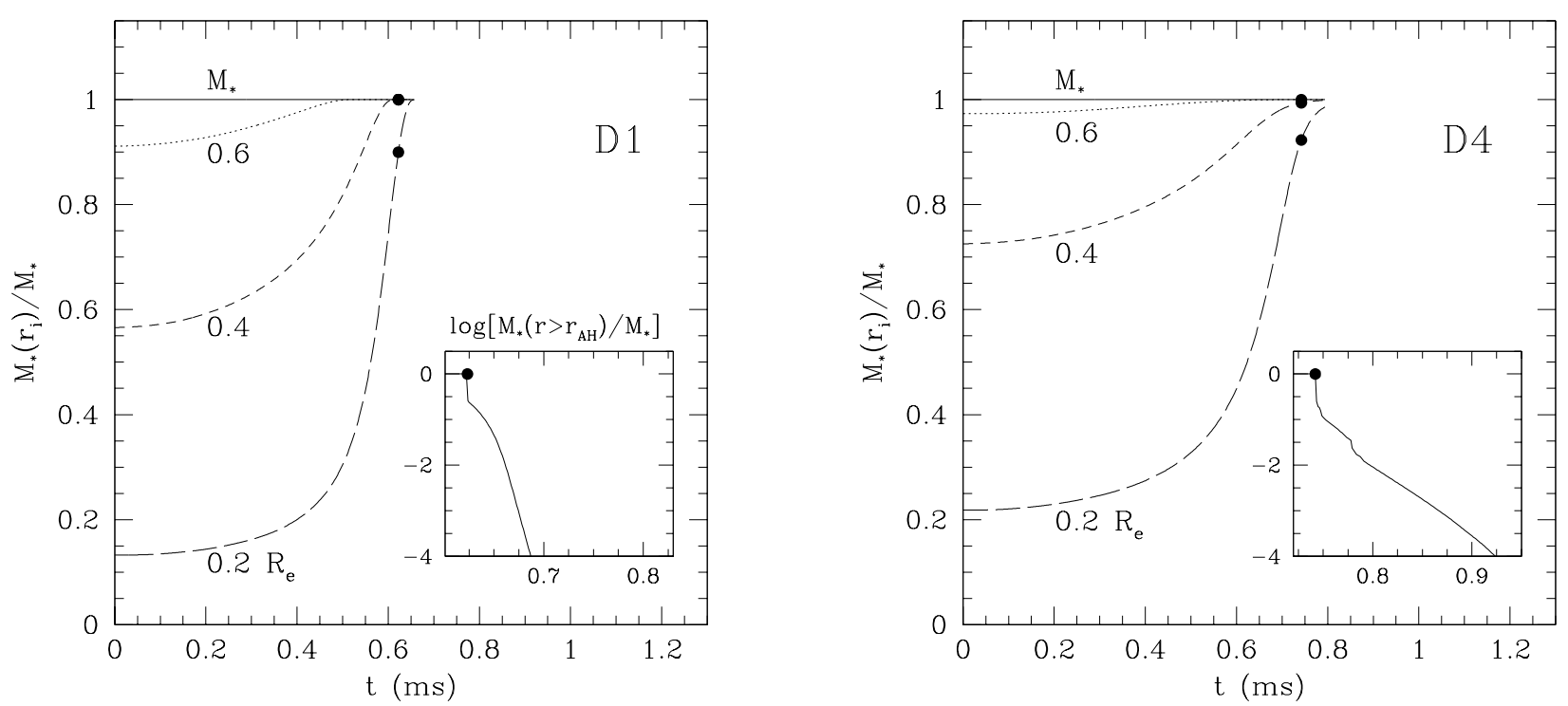

FIG. 9: Evolution of the mass fraction versus time during the collapse of D1 (left panel) and D4 (right panel). The rest mass is measured within two-spheres of coordinate radii $r_{i}=0.2,0.4$ and 0.6 times the initial stellar equatorial circumferential radius $R_{e}$ ( $c f$. Table $\mathbf{\square}$. Marked with filled dots on the different lines are the times at which the apparent horizon is first found (the data refers to a simulation with $96^{2} \times 48$ gridzones). The insets in both panels show, on a logarithmic scale, the evolution of the normalized rest mass outside the apparent horizon. Note that this is appreciably non-zero for a rather long time in case of model D4.

total time using a singularity excising region. Also in this case, we do not find evidence of shock formation nor of significant deviations from axisymmetry.

As mentioned in the Introduction, all simulations to-date agree that no massive and stable discs form for initial models of neutron stars that are uniformly rotating and when a polytropic EOS with $1 \leq N \leq 2$ is used. Our results corroborate this view and in turn imply that the collapse of a rapidly rotating old and cold neutron star cannot lead to the formation of the central engine believed to operate in a gamma-ray burst, namely a rotating black hole surrounded by a centrifugally-supported, self-gravitating torus. Relativistic simulations with more appropriate initial data, accounting in particular for the extended envelope of the massive progenitor star which is essential in the so-called collapsar model of gamma-ray bursts [87], will be necessary to shed light on the mechanism responsible for such events.

Convincing evidence has recently emerged [28] that a massive disc can be produced if the stellar models are initially rotating differentially and with initial total angular momenta $J / M^{2} \gtrsim 1$, as it may happen for young and hot neutron stars. In this case, the massive disc could emit intense gravitational radiation either through its oscillations [88] or as a result of the fragmentation produced by non-axisymmetric instabilities [28]. We are presently investigating this possibility and the results of our investigation will be reported in a forthcoming paper.

\section{Disc formation and differential rotation}

We now discuss in more detail two interesting properties of the matter dynamics in both slowly and rapidly rotating models: the evolution of the rest mass outside the apparent horizon and the development of differential rotation during the collapse.

In order to monitor the changes of the rest-mass distribution during the collapse we define the rest mass within a twosphere of coordinate radius $r_{i}<R_{e}$ as (see, for instance, [89])

$$
M_{*}\left(r_{i}\right)=\int_{r^{\prime}<r_{i}} \rho \alpha u^{0} \sqrt{\gamma} \mathrm{d}^{3} \mathbf{x}^{\prime}
$$

where $d^{3} \mathbf{x}^{\prime}$ is the $3 \mathrm{D}$ coordinate volume element. Shown in Fig.9 is the evolution of the rest masses measured within several representative two-spheres for models D1 (left panel) and D4 (right panel), respectively. Different lines refer to different coordinate radii for the two-spheres (i.e. $r_{i}=0.2,0.4$ and $0.6 R_{e}$, where $R_{e}$ is the initial equatorial circumferential radius) and are normalized to the total rest mass within the computational domain $M_{*}$, shown as a solid line. Marked instead with filled dots are the values of $M_{*}\left(r_{i}\right)$ at the times when the apparent horizon is first found; for simplicity, the data shown in Fig. 9 refer to a simulation with $96^{2} \times 48$ gridzones, but for this quantities higher resolutions have just the effect of shifting the time at which the apparent horizon is first found.

As mentioned before, the excised region is not introduced immediately after the apparent horizon has been found, but 

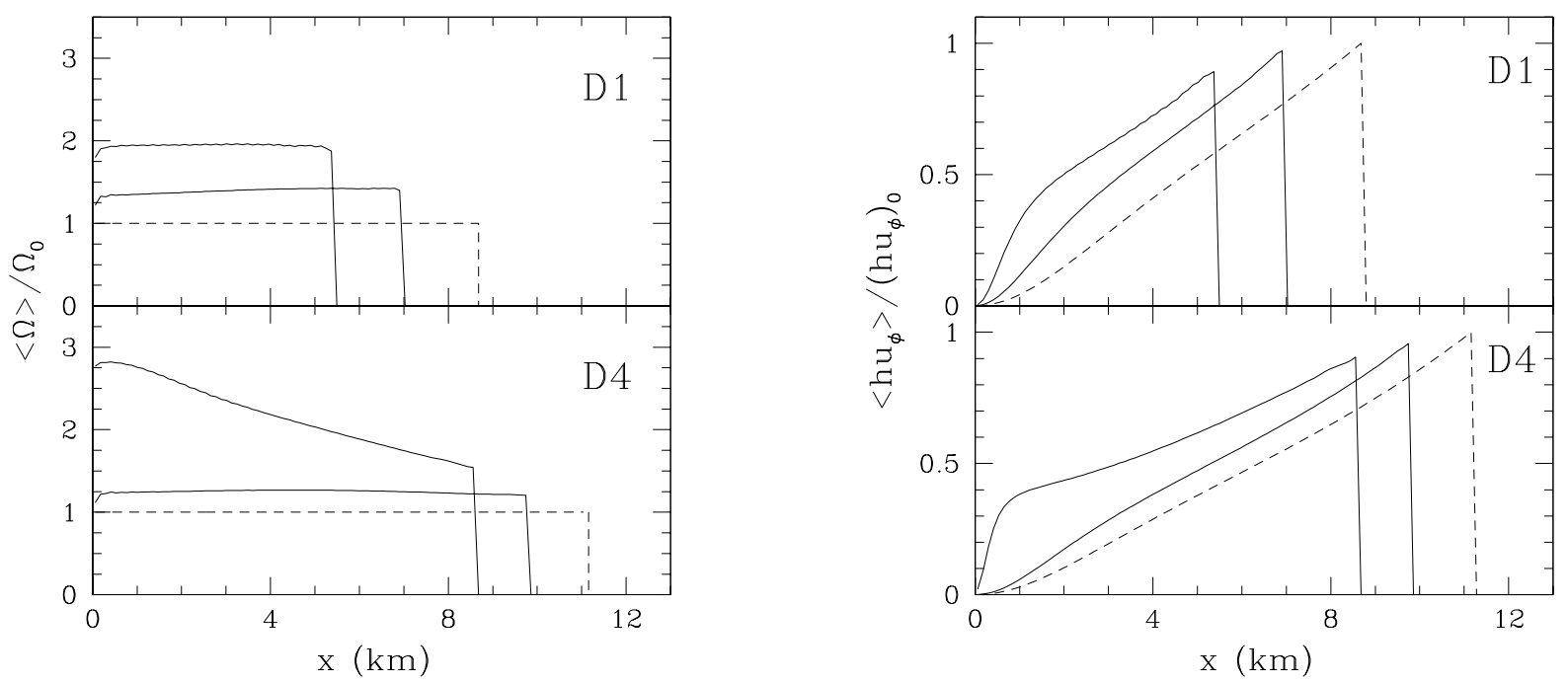

FIG. 10: Evolution of the averaged angular velocity $\langle\Omega\rangle$ (left panel) and of the averaged angular momentum per unit mass $\left\langle h u_{\phi}\right\rangle$ (right panel). Both quantities are measured at the stellar equator, are normalized to the initial value at the stellar surface and refer to both models D1 (upper parts) and D4 (lower parts).

only when this has grown to a sufficiently large size. When this happens, the inner part of the computational domain is removed and the integrals (4.1) are no longer meaningful. As a result, all of the curves in Fig. 9 are truncated at the time when the excision region is first introduced, which occurs at $t=0.72 \mathrm{~ms}$ and $t=0.79 \mathrm{~ms}$ for models $\mathrm{D} 1$ and $\mathrm{D} 4$, respectively.

A rapid comparison between the two panels of Fig. 9 is sufficient to identify the differences in the rest-mass evolution in slowly and rapidly rotating models. Firstly, the rest-mass distribution is very different already initially, being more uniform in D1 and more centrally concentrated in D4, as can be appreciated by comparing $M_{*}$ at $r_{i}=0.4 R_{e}$ and $0.6 R_{e}$. Secondly, the rest-mass infall is much faster for the slowly rotating model D1, while it is more progressive for model $\mathrm{D} 4$, as shown by the change in the fractional mass ratio at $r_{i}=0.4 R_{e}$. Finally, the amount of matter outside $r_{i}=0.4 R_{e}$ at the time when the apparent horizon is found, and which is very close to the amount of matter outside the apparent horizon, is different in the two cases, being essentially zero for model D1 and a few percent for model D4. A clearer view of this is presented in the two insets of Fig. 9 which show, on a logarithmic scale, the evolution of the normalized rest masses outside the apparent horizons, i.e. $M_{*}\left(r>r_{\mathrm{AH}}\right) / M_{*}$, since these first form and as they grow in time. It is interesting to note the different behaviour in this case with a rapid decrease when the rotation rate is small and a much slower one in the case of a rapidly rotating progenitor (Note that the two insets cover the same timescale although they refer to a different time interval.).

Two additional comments are worth making. The first one is that $M_{*}$ effectively includes also the rest mass in the atmosphere but this is always $\lesssim 10^{-4}$ of the total rest mass. The second one is that $M_{*}$ in Fig. 9 does not simply refer to the initial value of the total rest mass but is effectively computed at each step and appears constant in time because of the ability of the code to conserve rest mass. A closer look at the solid curve in Fig. 9 reveals, in fact, that $M_{*}$ varies over time to less than one part in $10^{4}$.

An interesting question to ask at this stage is whether these uniformly rotating models will develop any degree of differential rotation as the collapse proceeds. Part of the interest in this comes from the fact that neutron stars are thought to rotate differentially, at least during the initial stages of their life. This is expected to hold both when the neutron star is produced through a stellar core collapse, in which case the differential rotation may be present already in the stellar progenitor and is then amplified during collapse [90], and when the neutron star is the end-result of a binary merger of neutron stars [91]. However, as the neutron star cools and grows older, dissipative viscous effects or the coupling with non-turbulent magnetic fields are expected to bring the star into uniform rotation (see [92, 93, 94] for a detailed description of this process). It is therefore interesting to investigate whether a degree of differential rotation will be produced also during the final collapse of a uniformly rotating star to a Kerr black hole. To answer this question we have monitored both the averaged angular velocity $\langle\Omega\rangle$, defined as

$$
\langle\Omega\rangle \equiv \frac{1}{2}\left(\left.\frac{u^{\phi}}{u^{t}}\right|_{x \text {-axis }}+\left.\frac{u^{\phi}}{u^{t}}\right|_{y \text {-axis }}\right),
$$

and the corresponding averaged angular momentum per unit mass $\left\langle h u_{\phi}\right\rangle$, which is a conserved quantity along the path lines of fluid elements in an axisymmetric (but not necessarily stationary) spacetime [95]. Note that $u^{\phi} / u^{t}=\alpha v^{\phi}-\beta^{\phi}$ and the average over the two different directions is here used to compensate the small errors that are produced in the evaluation of 
these quantities near the axes.

We note that our measure of the differential rotation will depend on the specific slicing chosen. However, for the simulations reported here, the lengthscale of variation of the lapse function at any given time is always larger than the stellar radius at that time, ensuring that the events on the same timeslice are also close in proper time. A useful measure of the differential rotation that develops during collapse is the departure from unity of the ratio of the values of $\Omega$ at the centre and at the surface of the star on the equatorial plane and it is instructive to compare how this varies in the dynamics of the two models D1 and D4, which have been evolved using the same slicing.

The time evolution of $\langle\Omega\rangle$ and $\left\langle h u_{\phi}\right\rangle$ is presented in the two panels Fig. 10] whose lower parts refer to model D4 while the upper ones refer to model D1. Both quantities are shown normalized to their initial value at the stellar surface. Let us concentrate on the slowly rotating model first. The different lines refer to three representative times which are $t=0.0$ (shown as dashed), $t=0.45$ and $0.52 \mathrm{~ms}$, respectively. Initially, the angular velocity is, by construction, uniform throughout the star (left panel) and the corresponding specific angular momentum grows linearly with the distance from the stellar centre (right panel). As the collapse proceeds and the stellar size decreases, the angular velocity is expected to increase while the angular momentum per unit mass remains constant. This is indeed what happens for model D1, whose specific angular momentum is conserved with an overall error at the stellar surface which is always less than $10 \%$ and which decreases with resolution. A similar behaviour is observed also much later in the simulation, when the apparent horizon has been found and the singularity has been excised. Overall, the angular velocity in the collapsing model D1 grows like $\Omega(t) \propto r_{e}^{-n}$, where $n \simeq 1.5$ and therefore less than it would do in the case of the collapse of a Newtonian, uniform density star $($ i.e. $n=2)$; which is a result of relativistic and rotational effects (see [96]).

A comparison of the lower parts of the two panels in Fig. 10 is sufficient to realize that the evolution of the angular velocity is rather different for a rapidly rotating stellar model. The different lines in this case refer to $t=0.0,0.48$ and $0.65 \mathrm{~ms}$, respectively, and it is apparent that a non-negligible degree of differential rotation develops as the collapse proceeds, with a difference of a factor $\sim 2$ between the angular velocity of the inner and outer parts of the collapsing matter as the apparent horizon first appears. Clearly, this differential rotation is produced very rapidly and will persist only for a very short time before the star is enclosed in a trapped surface.

It is difficult to establish, at this stage, whether the differential rotation generated in this way could produce a phenomenology observable in some astrophysical context and more detailed investigations, in particular of the coupling of this differential rotation with magnetic fields [97, 98], are necessary. Finally, it is worth remarking that while differential rotation develops for model D4 but not for D1, the specific angular momentum is conserved to the same accuracy in both models.

\section{DYNAMICS OF THE HORIZONS}

In order to investigate the formation of a black hole in our simulations, we have used horizon finders, available through the Cactus framework, which compute both the apparent horizon and the event horizon. The apparent horizon, which is defined as the outermost closed surface on which all outgoing photons normal to the surface have zero expansion, is calculated at every time step and its location is used to set up the excised region inside the horizon. Specific technical details about the handling of the excised region for the fields are presented in [19, 22], while a brief discussion of how the hydrodynamical excision is performed in Whisky was presented in Section IIC

In contrast, the event horizon, which is an expanding null surface composed of photons which will eventually find themselves trapped, is computed a posteriori, once the simulation is finished, by reconstructing the full spacetime from the $3 \mathrm{D}$ data each simulation produces. In stationary black-hole systems, where no mass-energy falls into the black hole, the apparent and event horizons coincide, but generally (in dynamical spacetimes) the apparent horizon lies inside the event horizon. We have here used the fast solver of Thornburg [76] to locate the apparent horizon at every time step, and the levelset finder of Diener [99] to locate the event horizon after the simulation has been completed and the data produced is postprocessed.

In all cases considered, we have found that the event horizon rapidly grows to its asymptotic value after formation. With a temporal gap of $\sim 10 M$ after the formation of the event horizon, the apparent horizon is found and then it rapidly approaches the event horizon, always remaining within it. With the exception of the initial gap, the horizon proper areas as extracted from the apparent and event horizon are very close (see e.g., Fig. 16.

\section{A. Measuring the Event-Horizon Mass}

We measure the mass of the newly formed black hole to estimate the amount of energy that is emitted as gravitational radiation during the collapse. In particular, we do a simple energy accounting, comparing the mass of the black hole with the ADM mass of the spacetime computed by the initial data solver on a compactified grid extending to spatial infinity [81]. This value is slightly different ( $1 \%$ in the worst case) from the one which is instead computed on the finite domain of our computational grid at the initial time and after the constraints are solved. The difference between the two values can be used to define an "error-bar" for our measure of the black-hole mass and hence of the energy in gravitational waves ( $c f$. Fig. 15. Two notes are worth making about this error before we go on to discuss how the black-hole mass is actually measured. Firstly, the difference between the two masses represents the truncation error produced by the finite size of the computational domain and is conceptually distinct from the truncation error introduced by the finite differencing. While the first is assumed to be constant in time, the second in general grows 


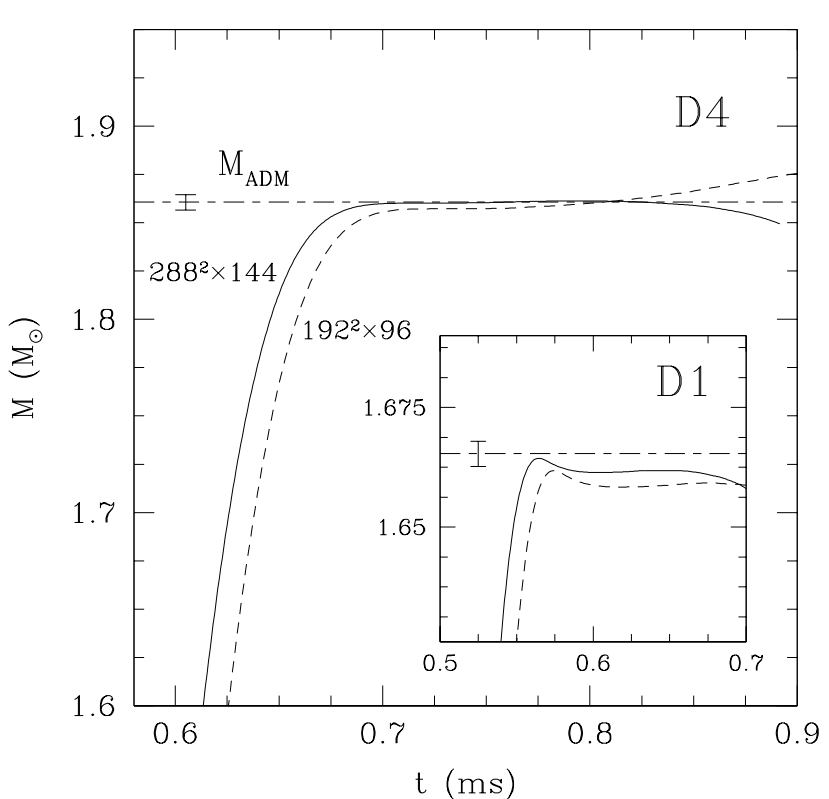

FIG. 11: Convergence of the measure of the black-hole mass as the resolution is increased. The curves refer to estimates using the eventhorizon equatorial circumference [i.e. eq. [5.1] ] and have been obtained using $288^{2} \times 144$ and $192^{2} \times 96$ zones, respectively. Shown in the small inset are the results for model D1, while those for model D4 are in the main panel.

with time (especially after the excision is made) and is monitored through the calculation of the constraint equations. Secondly, this error-bar sets a global lower limit on the accuracy of our measure of asymptotic quantities and therefore on the energy lost to gravitational waves during the collapse. No reliable measure of this lost energy can be made below the errorbar even if the constraint equations are solved to a larger precision (this will be discussed in more detail in Section VC.

The first and simplest method of approximating the blackhole mass is to note that, for a Kerr (or Schwarzschild) black hole, the mass can be found directly in terms of the eventhorizon geometry as

$$
M=\frac{C_{\mathrm{eq}}}{4 \pi},
$$

where $C_{\mathrm{eq}} \equiv \int_{0}^{2 \pi} \sqrt{g_{\phi \phi}} d \phi$ is the proper equatorial circumference. Provided there is a natural choice of equatorial plane, it is expected that, as the black hole settles down to Kerr, $C_{\text {eq }}$ will tend to the correct value. However, as numerical errors build up at late times it may be impossible to reach this asymptotic regime with sufficient accuracy.

The estimate of $M$ coming from the use of 5.1 is presented in Fig.11, which shows the time evolution of the eventhorizon equatorial circumference. The two lines refer to two different resolutions $\left(288^{2} \times 144\right.$ and $192^{2} \times 96$ zones, respectively) and should be compared with the value of the ADM mass $M_{\mathrm{ADM}}$ (indicated with a short-long dashed line), and with the error-bars as deduced from the initial data. Shown in

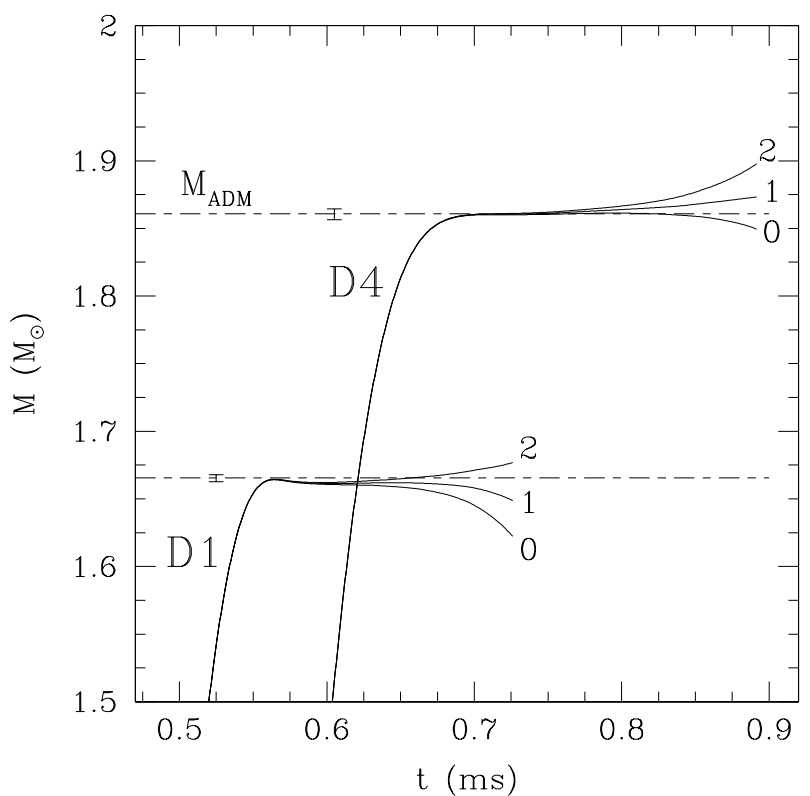

FIG. 12: Evolution of the event-horizon mass $M=C_{e q} / 4 \pi$ for models D1 and D4. Different lines refer to the different initial guesses for the null surface and are numbered 0,1 and 2 . Note that they converge exponentially to the correct event-horizon surface for decreasing times.

the small inset are the results for model D1, while those for model D4 are in the main panel.

Note that if a measure of the event horizon is not available, eq. 5.1 could be computed using the equatorial circumference of the apparent horizon (this is what was done, for instance, in [28]). Doing so would yield results that are similar to those shown in Fig. 11 although with a slightly larger deviation from $M_{\mathrm{ADM}}$. This is because we have found the apparent horizon to systematically underestimate the equatorial circumference. In particular, in the high-resolution run for model D4, the differences between the apparent and eventhorizon equatorial circumferences are $\lesssim 2 \%$.

Clearly, as the equatorial circumference grows, the agreement with the expected ADM mass improves as it does with the use of higher spatial resolution. However, equally evident is that the errors grow as the collapse proceeds and this is due, in part, to the loss of strict second-order convergence at later times, but also to the way the event horizon is found. The level-set approach of [99], in fact, needs initial guesses for the null surface, which converge exponentially to the correct event-horizon surface for decreasing times, hence introduces a systematic error in the calculation of the event horizon at late times. This is shown in Fig. 12, which presents the evolution of the event-horizon mass $M=C_{e q} / 4 \pi$ for models D1 and D4. Different lines refer to the different initial guesses and are numbered "0", "1" and "2", respectively (note that for the curves shown in Fig. 111 the initial guesses " 0 " and " 1 " were used for cases D4 and D1, respectively). 

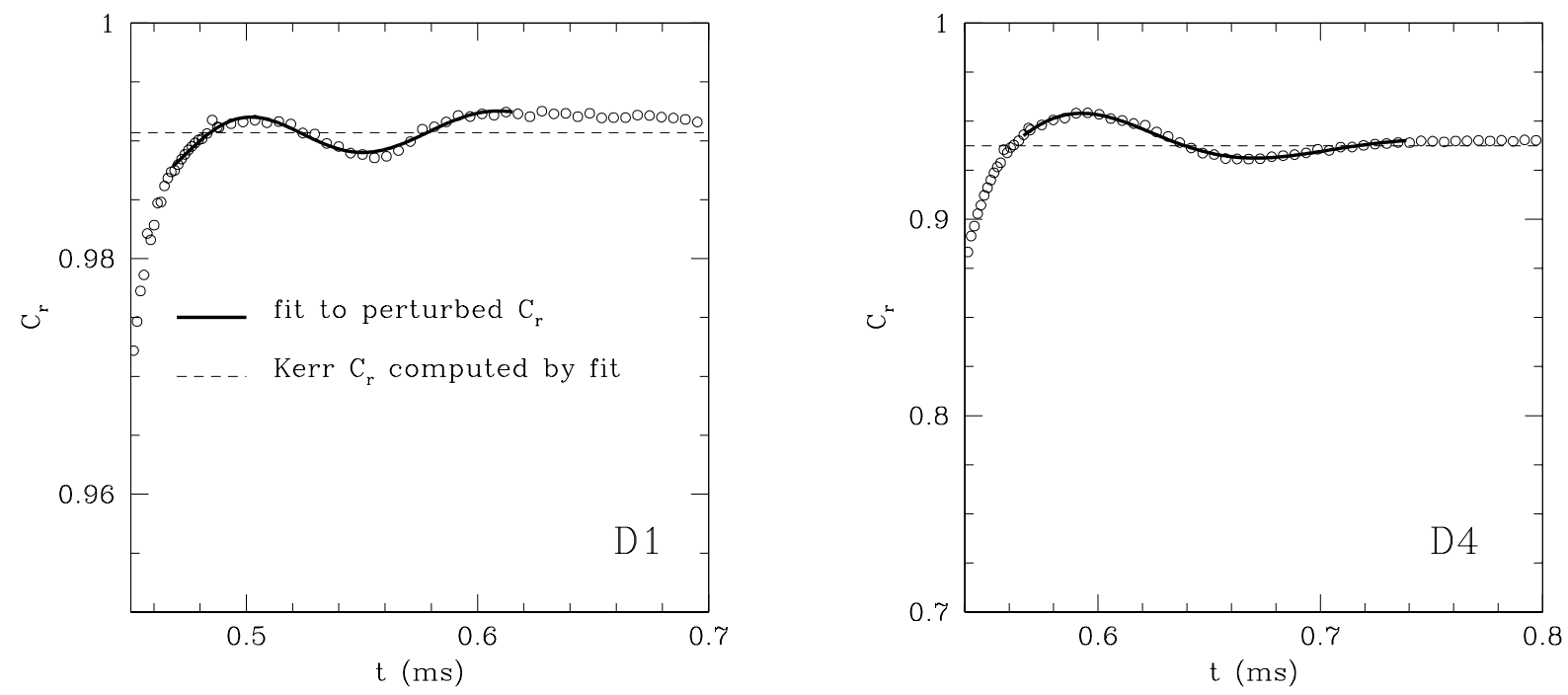

FIG. 13: Fitting the oblateness of the event horizon to QNMs of a Kerr black hole. The fit is shown with the solid line, while the open circles represent the computed values of $C_{r}$. The estimate for $C_{r}$ of a Kerr black hole having the fitted value of $a / M_{h o r}$ is shown with a dashed line.

\section{B. Measuring the angular momentum of the black hole}

A major difficulty in an accurate measurement of $M$ lies in the calculation of its non-irreducible part, i.e. in the part that is proportional to the black-hole angular momentum $J$. We now discuss a number of different ways to calculate $J$ from the present simulations; these measurements will then be used to obtain alternative estimates of $M$ in Section $\mathrm{VC}$

\section{Measuring J from the Horizon Distortion}

In a series of papers studying the dynamics of distorted black-hole spacetimes, it was shown that the horizon geometry provides a useful measure of the black-hole properties both in vacuum [100, 101, 102, 103] and when these are accreting matter axisymmetrically [104]. In particular, the idea is to look at the distortion of the horizon using the ratio of polar and equatorial proper circumferences, $C_{r} \equiv C_{\mathrm{pol}} / C_{\mathrm{eq}}$. For a perturbed Kerr black hole this is expected to oscillate around the asymptotic Kerr value with the form of a quasinormal mode (QNM). By fitting to this mode we extract an estimate of the angular momentum parameter $a / M_{h o r}$ from the relation [105]

$$
\frac{a}{M_{h o r}}=\sqrt{1-\left(-1.55+2.55 C_{r}\right)^{2}},
$$

where we have indicated with $M_{\text {hor }}$ the black-hole mass as measured from expression (5.2), which coincides with $M$ only if the spacetime has become axisymmetric and stationary. The fit through expression (5.2) is expected to be accurate to $\sim$ $2.5 \%$ [105].

The fit itself depends on an initial guess for $a / M_{h o r}$ and we start from a Schwarzschild black hole and iterate till the desired convergence is reached. This measure is not gauge invariant, although eq. (5.2) is independent of the spatial coordinates up to the definition of the circumferential planes, but works adequately with the gauges used here. The fit is best performed shortly after black-hole formation as the oscillations are rapidly damped. This minimizes numerical errors but in those cases where matter continues to be accreted, it may lead to inaccurate estimates of the angular momentum.

Examples of the fitting procedure are shown in Fig. 13 in which the fit is shown as a solid line, while the open circles represent the computed values of $C_{r}$; these are slightly noisy as a result of the interpolation needed by the level-set approach to find points on the horizon two-surface [99]. The estimate for $C_{r}$ of a Kerr black hole having the fitted value of $a / M_{h o r}$ is shown as a dashed line. Note that the values of $a / M_{\text {hor }}=0.21$ and $a / M_{\text {hor }}=0.54$ are very close to the total $J / M^{2}$ of the initial stellar models, i.e. 0.2064 and 0.5433 , as shown in Table This demonstrates that, to within numerical accuracy, the entire angular momentum of the spacetime ends up in the black hole.

Using expression (5.2) to estimate the angular momentum $J$ introduces an error, if the black hole has not yet settled to a Kerr solution. Having this in mind, however, it is possible to estimate the angular momentum as

$$
J=\left(\frac{a}{M_{h o r}}\right) M_{h o r} M \simeq\left(\frac{a}{M_{h o r}}\right) M^{2} .
$$

\section{Measuring $J$ with the dynamical-horizon framework}

A second method of approximating $J$ and hence measuring $M$ is to use the isolated and dynamical-horizon frameworks of Ashtekar and collaborators [106, 107, 108, 109, 110]. This assumes the existence of an axisymmetric Killing vector field 

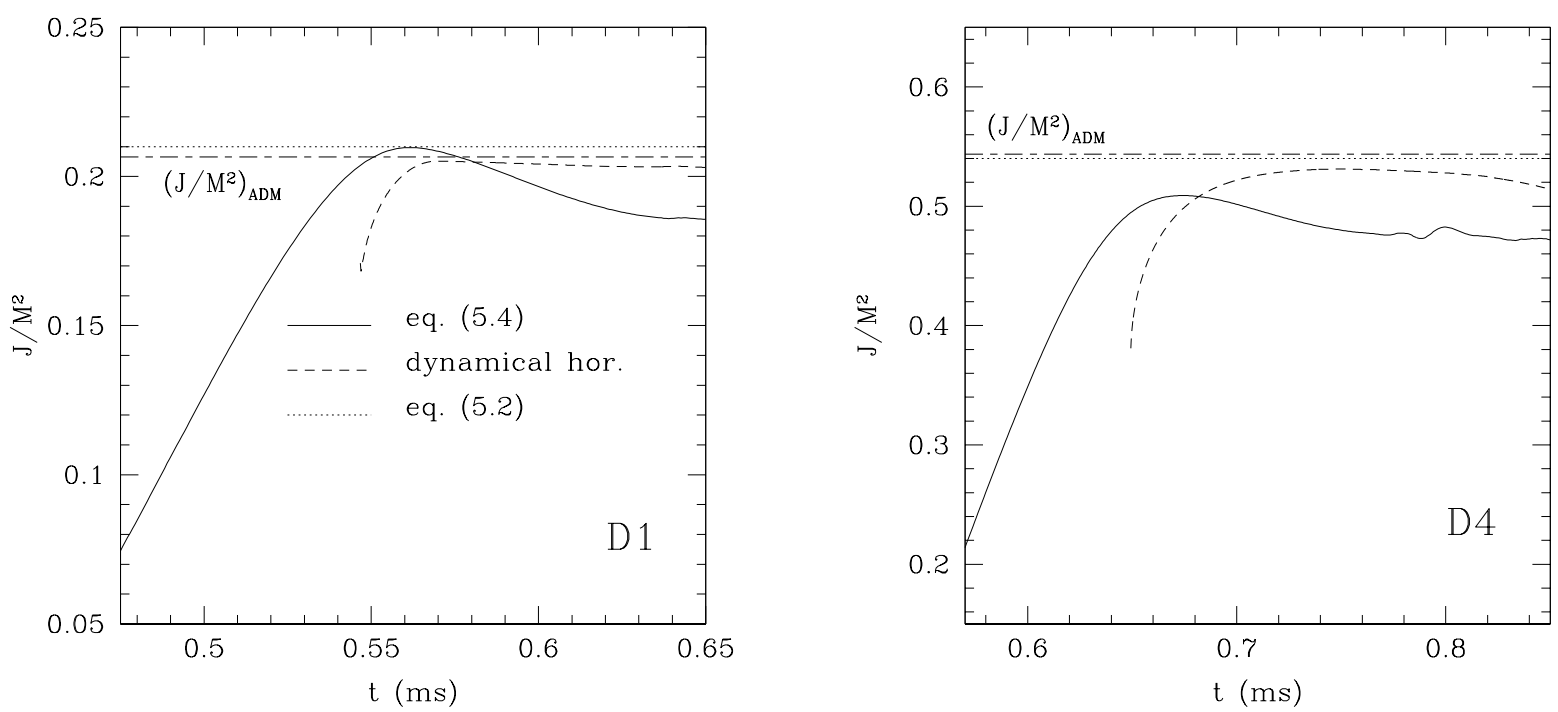

FIG. 14: Comparison of the different measures of the angular momentum for the cases D1 (left panel) and D4 (right panel). The estimate using the fit to the circumference ratio (see left panel of Fig. 13 is also shown. The dynamical-horizon spin measurement is considerably more accurate at late times as the event-horizon surfaces will diverge exponentially at this point. Shown with the horizontal short-long dashed lines are the values of $\left(J / M^{2}\right)_{\mathrm{ADM}}$ in the two cases as measured from the initial data (see main text for details).

TABLE II: Estimates of the black-hole angular momentum $J / M^{2}$ through the oblateness of the event horizon. The oscillations in the oblateness of the event horizon can be fitted to the normal modes of a Kerr black hole. Note that for each model the measured angular momentum is remarkably close to that of the initial spacetime $\left(J / M^{2}\right)$ ADM. Also reported are the initial ADM mass, the value of the equatorial circumference as obtained through the fit $\left(C_{r}\right)_{\mathrm{EH}}$, and the corresponding value obtained through the estimated spin parameter $\left(C_{r}\right)_{\text {Kerr }}$.

\begin{tabular}{cccccc}
\hline Model & $M_{\mathrm{ADM}}$ & $(J / M)_{\mathrm{ADM}}^{2}$ & $\left(J / M^{2}\right)_{\mathrm{EH}}$ & $\left(C_{r}\right)_{\mathrm{EH}}$ & $\left(C_{r}\right)_{\mathrm{Kerr}}$ \\
\hline D1 & 1.6653 & 0.2064 & 0.21 & 0.99 & 0.9916 \\
D2 & 1.7281 & 0.3625 & 0.36 & 0.97 & 0.9734 \\
D3 & 1.7966 & 0.4685 & 0.47 & 0.95 & 0.9544 \\
D4 & 1.8606 & 0.5433 & 0.54 & 0.94 & 0.9372 \\
\hline
\end{tabular}

intrinsic to a marginally trapped surface such as an apparent horizon. This gives an unambiguous definition of the spin of the black hole and hence of its total mass. If there is an energy flux across the horizon, the isolated-horizon framework needs to be extended to the dynamical-horizon formalism [109, 111].

In practice, our approach to the dynamical-horizon framework has been through the use of a code by Schnetter which implements the algorithm of [110] to calculate the horizon quantities. The advantage of the dynamical-horizon framework is that it gives a measure of mass and angular momentum which is accurately computed locally, without a global reconstruction of the spacetime. One possible disadvantage is that the horizon itself is required to be (close to) axisymmetric; so in case it deviates largely from axial symmetry, no informa- tion can be found. However, because arbitrarily large distortions are allowed as long as they are axisymmetric, we have not encountered problems in applying the dynamical-horizon framework to the horizons found in our simulations.

\section{Measuring J from the Angular Velocity of the Event Horizon}

A third method for computing $J$ only applies if an event horizon is found and if its angular velocity has been measured. In a Kerr background, in fact, the generators of the event horizon rotate with a constant angular velocity $\omega \equiv-g_{t \phi} / g_{\phi \phi}=$ $\sqrt{g_{t t} / g_{\phi \phi}}$. In this case the generator velocity can be directly related to the angular momentum parameter as

$$
\frac{a}{M}=\frac{J}{M^{2}}=\left[\frac{A \omega^{2}}{\pi}\left(1-\frac{A \omega^{2}}{4 \pi}\right)\right]^{1 / 2}
$$

As with the previous approximations, expression 5.4 is strictly valid only for a Kerr black hole and will therefore contain a systematic error which, however, decays rapidly as the black-hole perturbations are damped. On the other hand, the event horizon generator velocities have the considerable advantage that everything is measured instantaneously and the values of $\omega$ are valid whether or not the background is an isolated Kerr black hole. The disadvantage, though, is that, as mentioned above, the numerical event horizon surfaces become systematically less accurate at late times ( $c f$. Fig. [12). 


\section{Comparison of angular momentum measurements}

A detailed comparison of the three different methods for measuring the angular momentum of the black hole is shown in Fig. 14 The measurement of angular momentum using the angular velocity of the generators is shown as solid lines. Both for slowly (left panel) and rapidly (right panel) rotating stellar models, the event horizon has zero area (and thus zero angular momentum) when it is first formed. However, as the rotating matter collapses, the event horizon-area and angular momentum grow, the black hole is spun up and, to numerical accuracy, the total angular momentum of the spacetime is contained within the black hole ( $c f$. Fig.13). At late times, the estimate using the generator velocities of the event horizon drifts away, probably due to a combination of gauge effects and the systematic errors in the trial guesses for the null surfaces.

In the case of the slowly rotating model D1, in particular, the estimate from the dynamical-horizon finder is perfectly stable ( $c f$. dashed line in the left panel of Fig. 14, indicating that an approximately stationary Kerr black hole has been formed by the time the simulation is terminated. In the case of the rapidly rotating model $\mathrm{D} 4$, however, this is no longer the case as matter continues to be accreted also at later times, when the errors have also increased considerably. As a result, the measure of the spin through the dynamical-horizon finder is less accurate and does not seem to have stabilized by the time the simulation ends ( $c f$. dashed line in the right panel of Fig. 14. This may indicate that the final black hole has not settled down to a Kerr black hole on the timescales considered here.

\section{C. black-hole mass from the Christodoulou formula}

It was shown by Christodoulou that, in the axisymmetric and stationary spacetime of a Kerr black hole, the square of the black-hole mass $M$ is given by [112]

$$
M^{2}=M_{\mathrm{irr}}^{2}+\frac{4 \pi J^{2}}{A}=\frac{A}{16 \pi}+\frac{4 \pi J^{2}}{A},
$$

where $M_{\text {irr }}$ is the irreducible mass, $A$ is the event-horizon proper area, and $J$ is the black-hole angular momentum. As the black hole approaches a stationary state at late times, the apparent and event horizons will tend to coincide and in that case the mass of the black hole is very well approximated by the above formula.

We have applied the above formula, using the various methods for measuring the angular momentum $J$. In particular, using the method for obtaining $J$ from the distortion of the event horizon, through eq. 5.3], the black-hole mass is given by

$$
M^{2}=\frac{A}{8 \pi}\left(\frac{M_{h o r}}{a}\right)^{2}\left[1-\sqrt{1-\left(\frac{a}{M_{h o r}}\right)^{2}}\right] .
$$

If, on the other hand, $J$ is found from the angular velocity $\omega$ of the event horizon, then it is possible to use (5.4) in 5.5) and obtain

$$
M^{2}=\frac{A}{16 \pi-4 A \omega^{2}} .
$$

In the framework of dynamical horizons, expression 5.5 holds for any axisymmetric isolated or dynamical horizon, independently of whether it is stationary.

Figure 15 collects the four different ways of measuring the black-hole mass for the collapse of models D1 and D4. The different lines refer to the different approaches we have outlined above. In addition, we display the irreducible mass $M_{\text {irr }}$. The left panel of Fig. 15 in particular, shows the results of the different measures for the slowly rotating model D1. Because in this case all of the matter rapidly collapses into the black hole, the different estimates of the total mass agree very well. However, already in this slowly rotating case the irreducible mass of the apparent horizon is noticeably lower. The left panel also shows that while the different methods provide comparable estimates, only the one corresponding to eq. 5.1. (i.e. the solid line) falls for some time within the error-bar provided by the initial estimate of $M_{\mathrm{ADM}}$ (this is particularly evident in the inset). Because when this happens the norms of the Hamiltonian constraint have not yet started to grow exponentially and the largest value of the constraint violation is about an order of magnitude smaller (i.e. the $\mathrm{L}_{1}$ norm of the Hamiltonian constraint is $\sim 4.9 \times 10^{-4}$ at $t=0.56 \mathrm{~ms}$ ) we can use the error-bar in $M_{\mathrm{ADM}}$ to place an upper bound of $0.5 \% M_{\mathrm{ADM}}$ to the energy lost through the emission of gravitational radiation in this case. Clearly, the true bound is certainly considerably lower and we expect that with accuracies comparable to the ones of 2D simulations, our estimates of the efficiency of gravitational radiation emission could converge to the values of Stark and Piran [32].

The right panel of Fig. [15] on the other hand, shows the results of the different mass measures for the rapidly rotating model D4. In this case, the contribution from the spin energy is considerably larger and noticeable differences appear among the different approaches. Since all seem to have systematic errors, this makes it less trivial to establish which method is to prefer. On one hand, those methods using information from the event-horizon equatorial circumference or that fit the perturbations of the event horizon [i.e. eqs. 5.1] and (5.6)] seem to provide accurate estimates at earlier times but suffer of the overall inaccuracy at later stages, when the initial guesses for the null surface are distinct. It is indeed at these early times that these measurements are within the error-bar provided by the initial estimate of $M_{\mathrm{ADM}}$. On the other hand, those methods that measure the angular velocity of the null generators [i.e. eq. [5.7] or that use the dynamicalhorizon framework, produce reasonably accurate estimates, that converge with resolution, that monotonically grow in time and that are within the error-bar of the initial estimate of $M_{\mathrm{ADM}}$. Furthermore, in the case of the dynamical-horizon framework, this is not only physically expected, given that a small but non-zero fraction of the matter continues to be accreted nearly until the end of the simulation, but it is also guaranteed analytically.

Because of these differences in the measures of $M$ and because the black hole does not have time to settle down to a 

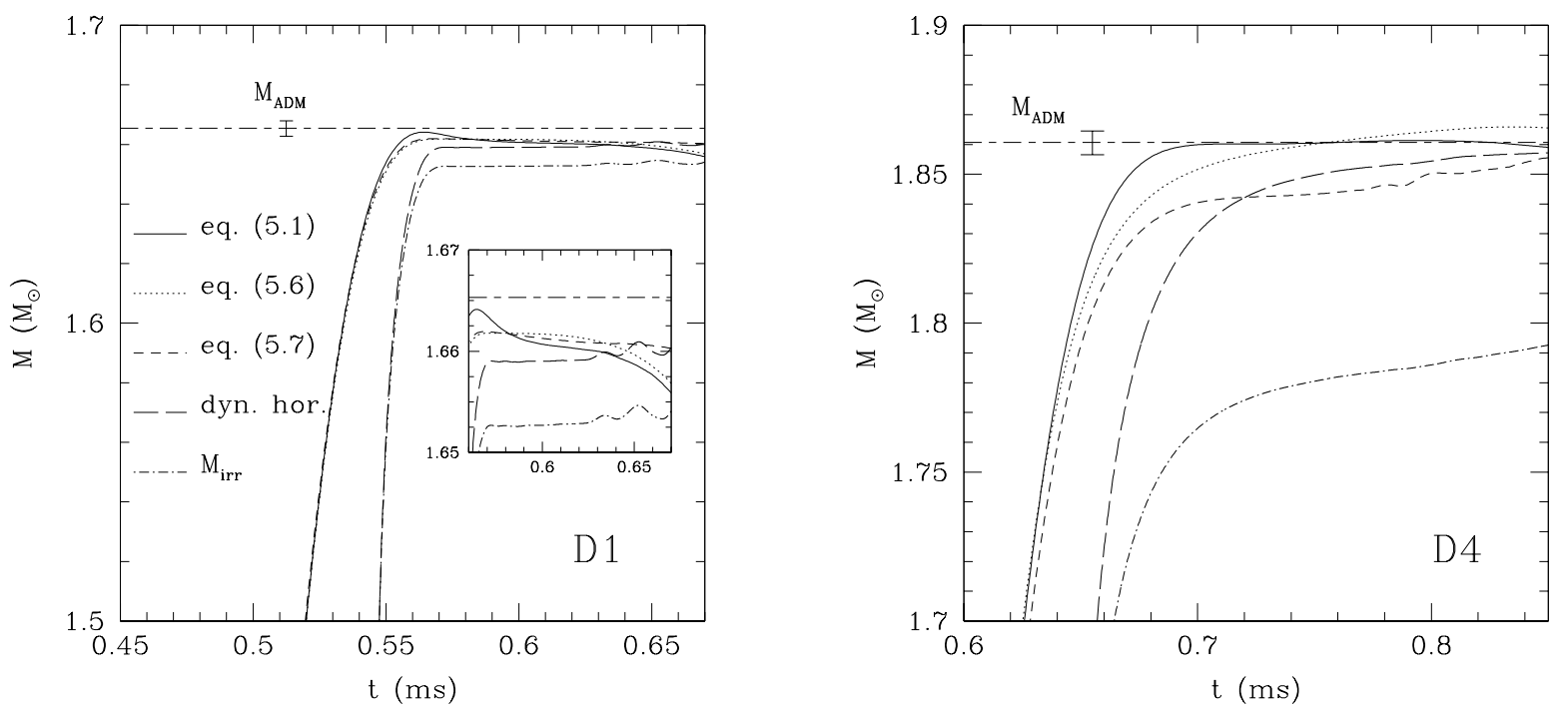

FIG. 15: Comparison of the five different approaches used in the measure of the total black-hole mass for the collapse of models D1 and D4. Different lines refer to the different methods discussed in the main text. The left panel (model D1), shows that the different methods are overall comparable when the rotation is slow, but that differences are already present (this is as shown in the inset). The right panel (model D4) shows that the different measures can be considerably different when the rotation is large.

constant total mass, the upper bound on the energy emission is more conservative than in case D1. In particular, taking again as a reference the time when the estimate relative to eq. 5.1) is within the error-bar (i.e. at $t=0.70 \mathrm{~ms}$ ) and the largest value of the constraint violation is about an order of magnitude smaller (i.e. the $\mathrm{L}_{1}$ norm of the Hamiltonian constraint is $\sim 1.2 \times 10^{-3}$ ) and is not yet growing exponentially, we place an upper bound of $1 \% M_{\mathrm{ADM}}$ on the energy lost through gravitational radiation. Once again, we expect the true value to be considerably smaller.

One obvious and expected result is that the irreducible mass in the collapse of model D4 (the dot-dashed line in the right panel of Fig. 15 deviates by a large amount from the actual black hole mass, since it does not include the rotational energy of the black hole.

Finally, we will make a comment on the different methods used for measuring the mass and spin of a black hole in a numerical simulation. Although the direct comparison of many different methods employed here have provided valuable information on the dynamics of the system, we have found the dynamical-horizon framework to be simple to implement, accurate and not particularly affected by the errors from which equivalent approaches seem to suffer, as shown in our Figs. 15 and 14 As a result, we recommend its use as a standard tool in numerical relativity simulations.

\section{Reconstructing the global spacetime}

All of the results presented and discussed in the previous Sections describe only a small portion of the spacetime which has been solved during the collapse. In addition to this, it is interesting and instructive to collect all of these pieces of in- formation into a global description of the spacetime and look for those features which mark the difference between the collapse of slowly and rapidly rotating stellar models. As we discuss below, these features emerge in a very transparent way within a global view of the spacetime.

To construct this view, we use the worldlines of the most representative surfaces during the collapse, namely those of the equatorial stellar surface, of the apparent horizon and of the event horizon. For all of them we need to use properly defined quantities and, in particular, circumferential radii. The results of this spacetime reconstruction are shown in Fig.16 whose left and right panels refer to the collapse of models D1 and D4, respectively. The different lines indicate the worldlines of the circumferential radius of the stellar surface (dotted line), as well as of the apparent horizon (dashed line) and of the event horizon (solid line). Note that for the horizons we show both the equatorial and the polar circumferential radii, with the latter being always smaller than the former. For the stellar surface, on the other hand, we show the equatorial circumferential radius only. This is because the calculation of the stellar polar circumferential radius requires a line integral along the stellar surface on a given polar slice. Along this contour one must use a line element which is suitably fitted to the stellar surface and diagonalized (see [103] for a detailed discussion). In the case of model D4, however, this is difficult to compute at late times, when the disc is formed and the line integral becomes inaccurate.

Note that in both panels of Fig. 16 the event horizon grows from an essentially zero size to its asymptotic value. In contrast, the apparent horizon grows from an initially non-zero size and, as it should, is always contained within the event horizon. At late times, the worldlines merge to the precision at which we can compute them. A rapid look at the two pan- 

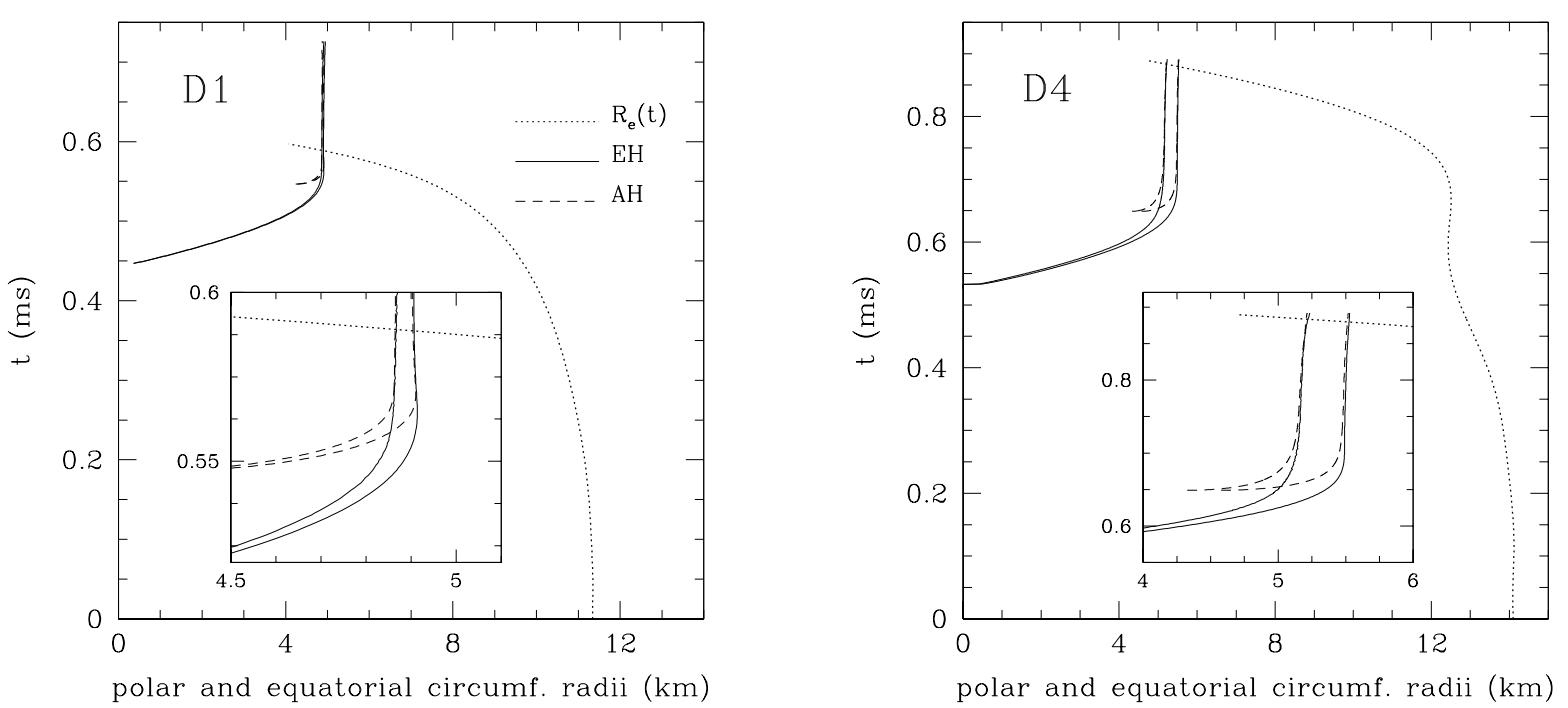

FIG. 16: Evolution of the most relevant surfaces during the collapse for the cases D1 and D4. Solid, dashed and dotted lines represent the worldlines of the circumferential radii of the event horizon, of the apparent horizon and of the stellar surface, respectively. Note that for the horizons we plot both the equatorial and the polar circumferential radii, while only the equatorial circumferential radius is shown for the stellar surface. Shown in the insets are the magnified views of the worldlines during the final stages of the collapse.

els of Fig.16 is sufficient to appreciate the different properties in the dynamics of the collapse of slowly and rapidly rotating models.

Firstly, in the case of model D1, the differences between the equatorial and polar circumferential radii of the two trapped surfaces are very small and emerge only in the inset that offers a magnified view of the worldlines during the final stages of the collapse. This is not the case for model D4, for which the differences are much more evident and can be appreciated also in the main panel. Of course, this is what one expects given that the ratio of these two quantities depends on $a / M$ and is $\sim 1$ for a slowly rotating black hole ( $c f$. Table $\Pi$.

Secondly, the worldlines of the stellar equatorial circumferential radius are very different in the two cases. In the slowly rotating model D1, in particular, the star collapses smoothly and the worldline always has negative slope, thus reaching progressively smaller radii as the evolution proceeds ( $c f$. left panel of Fig. 16. By time $t \simeq 0.59 \mathrm{~ms}$, the stellar equatorial circumferential radius has shrunk below the corresponding value of the event horizon. In the case of the rapidly rotating model D4, on the other hand, this is no longer true and after an initial phase which is similar to the one described for D1, the worldline does not reach smaller radii. Rather, the stellar surface slows its inward motion and, at around $t \sim 0.6$ $\mathrm{ms}$, the stellar equatorial circumferential radius does not vary appreciably. Indeed, the right panel of Fig. 16 shows that at this stage the stellar surface moves to slightly larger radii. This behaviour marks the phase in which a flattened configuration has been produced and the material at the outer edge of the disc experiences a stall ( $c f$. the middle and lower panels of Fig. (5). As the collapse proceeds, however, also this material will not be able to sustain its orbital motion and, after $t \sim 0.7$ $\mathrm{ms}$, the worldline moves to smaller radii again. By a time $t \simeq 0.9 \mathrm{~ms}$, the stellar equatorial circumferential radius has shrunk below the corresponding value of the event horizon.

\section{CONCLUSION}

Although 3D numerical relativity has been a very active research area for several years now, there are still a number of technical issues to be addressed and physical problems to be investigated in detail. Separate progress has been made so far in obtaining long-term stable evolutions of vacuum spacetimes and of spacetimes with matter. Both of them have posed significant numerical problems because of the existence of horizons containing physical singularities, in one case, and the development of non-linear hydrodynamical phenomena such as shocks, in the other. In black-hole vacuum spacetimes, these problems have successfully been dealt with by using better suited formulations of the Einstein equations and by employing excision techniques for the regions of the spacetime containing the singularity. In spacetimes containing matter, on the other hand, sophisticated numerical techniques (such as the HRSC methods) have been employed to accurately track the dynamics of the shocks.

Here, we have combined these two different approaches by implementing excision techniques within a forming horizon, thus following the dynamics of the matter as it accretes onto the developing black hole. We have shown that doing so allows the numerical evolution to proceed uninhibited from fully regular initial conditions of matter in equilibrium and devoid of trapped surfaces, up to a vacuum spacetime featuring an event horizon enclosing an excised physical singularity. This new important ability in numerical relativity evolutions will help in a more detailed investigation of complex astro- 
physical systems, such as the coalescence of neutron star binaries, considered as a prime candidate for the detection of gravitational waves, and of the collapse of stellar cores, considered as the progenitors of gamma-ray bursts.

Our hydrodynamical excision technique is implemented within a new 3D general-relativistic numerical evolution code that combines state-of-the-art numerical methods for the spacetime evolution (i.e. the NOK formulation of the Einstein equations with Gamma-driver shift conditions) with an accurate hydrodynamical evolution employing several high-order HRSC methods. The evolution of the spacetime and of the hydrodynamics is coupled transparently through the method of lines, which allows for the straightforward implementation of various different time-integrators.

As a first astrophysical problem for this novel setup, we have here focused on the collapse of rapidly rotating relativistic stars to Kerr black holes. The stars are assumed to be in uniform rotation and dynamically unstable to axisymmetric perturbations. While the collapse of slowly rotating initial models proceeds with the matter remaining nearly uniformly rotating, the dynamics is shown to be very different in the case of initial models rotating near the mass-shedding limit, for which strong differential rotation develops. Although the stars become highly flattened during collapse, attaining a disc-like shape, the collapse cannot be halted because the specific angular momentum is not sufficient for a stable disc to form. Instead, the matter in the disc spirals towards the black hole and angular momentum is transferred inward to produce a spinning black hole.

Several different approaches have been employed to compute the mass and angular momentum of the newly formed Kerr black hole. Besides more traditional methods involving the measure of the geometrical properties of the apparent and event horizons, we have fitted the oscillations of the perturbed Kerr black hole to specific quasi-normal modes obtained by linear perturbation theory. In addition, we have also considered the recently proposed isolated and dynamical horizon frameworks, finding it to be simple to implement and yielding estimates which are accurate and more robust than those of other methods. This variety of approaches has allowed for the determination of both the mass and angular momentum of the black hole with an accuracy unprecedented for a 3D simulation. These measures, in turn, have allowed us to set upper limits on the energy and angular momentum that could be lost during the collapse in the form of gravitational radiation.

Work using mesh-refinement techniques is already in progress to extract more precise information and waveforms for the gravitational radiation recorded at large distances from the collapsing stars. This aspect of the gravitational collapse has not yet been considered in full 3D simulations and will be reported in a forthcoming paper [45]. Finally, all of the techniques discussed here will also be applied to the study of the collapse of differentially rotating stars, governed by more realistic and non-isentropic EOSs. The expectation is that initial data with $J / M^{2} \gtrsim 1$ can be constructed in this case, whose collapse could lead to the formation of a massive disc orbiting around the newly formed Kerr black hole [12, 28, 38].

\section{APPENDIX A: NUMERICAL METHODS}

In this Appendix we focus on the numerical methods that the Whisky code incorporates for the solution of the general relativistic hydrodynamics equations. The corresponding methods for the spacetime equations are those implemented in the Cactus code and they have been reported elsewhere and the interested reader is addressed to [13, 57] for more details.

As mentioned in the main text, our code uses highresolution shock-capturing methods based on reconstruction evolution methods. In this approach, all variables $\mathbf{q}$ are represented on the numerical grid by cell-integral averages. The function is then reconstructed within each cell, usually by piecewise polynomials in a way that preserves conservation of the variables q. This gives two values at each cell boundary which are then used to solve (approximately) the Riemann problem, giving the flux through the cell boundary. A Method of Lines approach is then used to update in time. We will here give brief descriptions of each method, but further details can be found in [39].

\section{Time update: the method of lines}

The reconstruction methods guarantee that a prescribed order of accuracy is retained in space. However, the need to retain a high-order accuracy also in time can complicate considerably the evolution from a time-level to the following one. As a way to handle this efficiently, we have chosen to follow a MoL approach [73, 74]. Here, the continuum equations are considered to be discretized in space only. The resulting system of ordinary differential equations (ODEs) can then be solved numerically with any stable solver. This method minimizes the coupling between the spacetime and hydrodynamics solvers and allows for a transparent implementation of different evolution schemes.

MoL itself does not have a precise truncation error but, rather, it acquires the truncation order of the time-integrator employed. Several integrators are available in our implementation of MoL, including the second-order Iterative Crank Nicholson (ICN) solver and Runge-Kutta (RK) solvers of first to fourth-order accuracy. The second and third-order RK solvers are known to be TVD whilst the fourth-order is known to not be TVD [113, 114]. As the coupling between the spacetime and the hydrodynamics is only second-order accurate, we typically use the ICN solver.

The calculation of the right hand side to feed to the ODE splits into the following parts:

1. Calculation of the source terms $\mathbf{s}\left(\mathbf{q}\left(x_{j_{1}}^{(1)}, x_{j_{2}}^{(2)}, x_{j_{3}}^{(3)}\right)\right)$ at all the grid points.

2. For each direction $x^{(i)}$ :

- Reconstruction of the data $\mathbf{q}$ to both sides of a cell boundary. In this way, two values $\mathbf{q}_{L}$ and $\mathbf{q}_{R}$ of $\mathbf{q}_{j_{i}+1 / 2}$ are determined at the cell boundary; $\mathbf{q}_{L}$ is obtained from cell $j_{i}$ (left cell) and $\mathbf{q}_{R}$ from 
cell $j_{i}+1$ (right cell) (see Appendix A2 for more details).

- Solution at cell boundary of the approximate Riemann problem having the values $\mathbf{q}_{L, R}$ as initial data (see Appendix A3 for more details).

- Calculation of the inter-cell flux $\mathbf{f}^{\left(x^{(i)}\right)}\left(\mathbf{q}_{j_{i}+1 / 2}\right)$, that is, of the flux across the boundary between a cell (e.g., the $j_{i}$-th) and its closest neighbour (e.g., the $\left(j_{i}+1\right)$-th).

3. Recovery of the primitive variables and computation of the stress-energy tensor for use in the Einstein equations.

As a result of steps 1.-3., the core of the Whisky code is effectively represented by two routines. One that reconstructs the function $\mathbf{q}$ at the boundaries of a computational cell and another one that calculates the inter-cell flux $f$ at this cell boundary.

\section{Reconstruction methods}

For the reconstruction procedure we have implemented several different approaches, including slope-limited TVD methods, the Piecewise Parabolic Method [48] and the Essentially Non-Oscillatory method [49].

The TVD method uses limiters to avoid oscillations at shocks: we typically use the Van Leer monotized centred method, although a variety of others (minmod, Superbee) have also been implemented. This method is simple and computationally the least expensive to implement, but is at most second-order accurate, dropping to first-order at local extrema.

The PPM method of Colella and Woodward [48] is a composite reconstruction method that has special treatments for shocks, where the reconstruction is modified to retain monotonicity, and contact surfaces, where the reconstruction is modified to sharpen the jump. PPM contains a number of tunable parameters, but we always use those suggested by [48]. PPM is third-order accurate at most.

The ENO methods have a large number of variants, with the common theme that the "least oscillatory" stencil amongst all possible stencils of a given order is chosen. In practice we use it in its simplest form: direct piecewise polynomial reconstruction of the variables, as described in [115]. ENO has no tunable parameters besides the order of accuracy, which may be arbitrary.

All these methods are stable in the presence of shocks. By default we use PPM as this seems to be the best balance between accuracy and computational efficiency, as shown, for example, in [84], and is our best choice for all the test evolutions we present in Appendix B

\section{Approximate Riemann problem solvers}

Once a reconstruction procedure has provided data on either side of each cell boundary, this is then used to specify the initial states of the semi-infinite piecewise constant Riemann problems. Since the exact solution of the Riemann problem [116] is still too costly to use, even when recast in an efficient form [117, 118], we have here implemented three different approximate Riemann solvers.

The first and simplest method implemented is the HLLE method [50]. This approximates the solution by only two waves with the intermediate state given by the conservation of the mass-flux. This method is very efficient but diffusive. The second method is the Roe solver [119]. This solves a linearized problem at each boundary, approximating every wave by either a shock or a contact discontinuity. This method is less efficient but very accurate. However, it may have problems near sonic points. The third method is the Marquina solver [120, 121]. This is similar to the Roe solver, except that at possible sonic points a Lax-Friedrichs flux (analogous to the HLLE method) is used, ensuring that the solution does not contain rarefaction shocks. Note that we use the modified method of [51] instead of the original method.

Both the Roe and Marquina solvers require the computation of the eigenvalues and eigenvectors (from both the right and left cell) of the linearized Jacobian matrices $\mathbf{A}_{L}$ and $\mathbf{A}_{R}$ given by $\mathbf{f}_{L}=\mathbf{A}_{L} \mathbf{q}_{L}$ and $\mathbf{f}_{R}=\mathbf{A}_{R} \mathbf{q}_{R}$. We use an implemention of the analytic expression for the left eigenvectors [52], thus avoiding the computationally expensive inversion of the three $5 \times 5$ matrices of the right eigenvectors, associated to each spatial direction. We also use a compact version of the flux formula (a variant on the methods described in [53]) to increase speed and accuracy. These improvements bring a $\sim 40 \%$ reduction of the computational time spent in the solution of the hydrodynamics equations and $\mathrm{a} \sim 5-10 \%$ reduction in evolutions involving also the time integration of the Einstein equations. The small overall gain in efficiency is due to the fact that only around half of the computational time is spent computing the update of the hydrodynamic variables, with the other half spent in the update of the spacetime field variables. Of the time spent computing the hydrodynamic update terms around one third is spent solving the Riemann problem. These improvements were made by Joachim Frieben at the Universidad de Valencia.

\section{Treatment of the atmosphere}

At least mathematically, the region outside our initial stellar models is assumed to be perfect vacuum. Independently of whether this represents a physically realistic description of a compact star, the vacuum represents a singular limit of the equations 2.10 and must be treated artificially. We have here followed a standard approach in computational fluiddynamics and added a tenuous "atmosphere" filling the computational domain outside the star. This approach, which was implemented also in [14,91], was instead not used in the simulations presented in [122], where new strategies were sug- 
gested. Unfortunately, these corrections would not have been effective here because of the conservation form of our hydrodynamical equations (2.10), which we have used to guarantee the correct evolution of shocks.

We have treated the atmosphere as a perfect fluid governed by the same polytropic EOS used for the bulk matter, but having a zero coordinate velocity. Furthermore, the rest-mass density is several (usually 7) orders of magnitude smaller than the initial central density. Note that the atmosphere used for the calculation of the initial data and the one evolved during the simulations need not be the same. Indeed, for the initial stellar models used for the collapse calculations we have typically set the atmosphere to be two orders of magnitude smaller than the evolved one to minimise spurious matter accretion onto the black hole. In the pulsation tests presented in Appendix $\mathrm{B}$ on the other hand, the initial and evolved atmospheres are the same.

The evolution of the hydrodynamical equations in gridzones where the atmosphere is present is the same as the one used in the bulk of the flow. Furthermore, when the rest mass in a gridzone falls below the threshold set by the atmosphere, that gridzone is simply not updated in time.

As mentioned in Section IVB the use of a tenuous atmosphere has no dynamical impact and does not produce any increase of the mass of the black hole that can be appreciated in our simulations. With the rest-mass densities used here for the atmosphere, in fact, and using the mass accretion rates measured once the apparent surface is first found, we have estimated that a net increase of $\sim 1 \%$ in the black-hole mass would require an integration time of $\sim 10^{4} \mathrm{M}$. These systematic errors are well below our truncation errors, even at the highest resolutions we can afford.

\section{APPENDIX B: NUMERICAL TESTS}

Several tests have been performed to assess the stability and accuracy of our code, some of which have also provided new interesting physical results. Here, we briefly discuss some of the most representative of these tests and results, referring the interested reader to [39] for more details.

First of all, we consider a standard shock-tube test, setting as initial data a global Riemann problem, in particular one in which the initial discontinuity is orthogonal to the main diagonal of our cubic grid. More precisely the initial data consists of a left $(L)$ and right $(R)$ states with values given respectively by

$$
\begin{array}{lll}
\rho_{R}=1 ; & p_{R}=1.666 \times 10^{-6} ; & v_{R}=0, \\
\rho_{L}=10 ; & p_{L}=13.333 ; & v_{L}=0 .
\end{array}
$$

In Fig. 17we show the solution at a given time together with the exact solution. The excellent agreement of the two sets of curves is particularly remarkable if one bears in mind that the evolution is fully $3 \mathrm{D}$ and not simply along a coordinate axis.

Next, we consider the evolution of a stable relativistic polytropic spherical (TOV) star. As this is a static solution, no evolution is expected. Yet as shown in Fig. 18, both a small

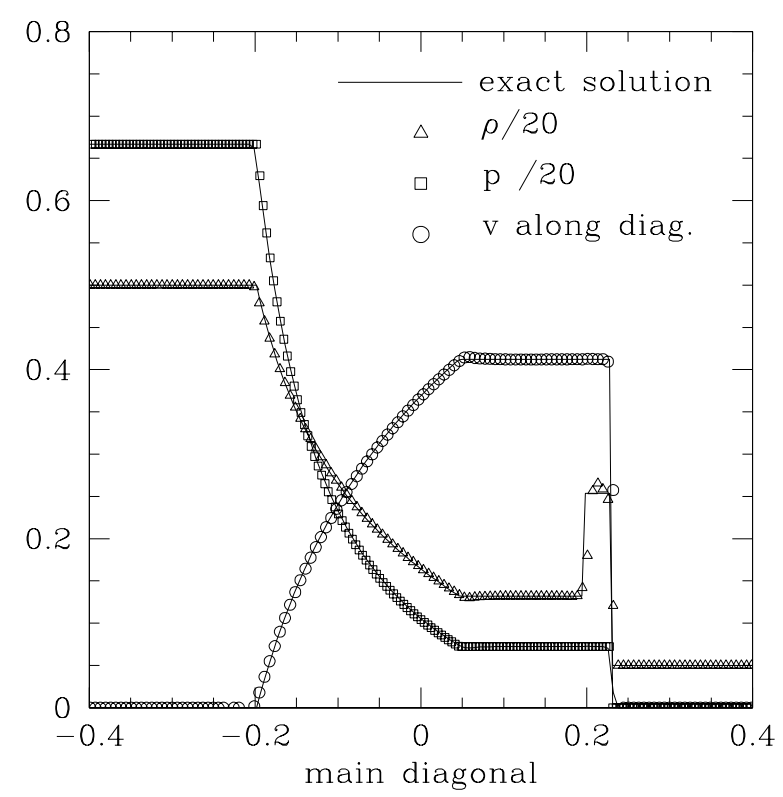

FIG. 17: Solution of a Riemann problem set on the main diagonal of the cubic grid. The figure shows the comparison of the hydrodynamical variables evolved by Whisky, indicated with symbols, with the exact solution. The numerical simulation was obtained with the van Leer reconstruction method and the Roe solver, on a grid with $140^{3}$ points.

periodic oscillation and a small secular increase of the central density of the star are detected during the numerical evolution of the equations. Both effects have, however, a single explanation. Since the initial data contains also a small truncation error, this is responsible for triggering radial oscillations which appear as periodic variations in the central density. As the resolution is increased, the truncation error is reduced and so is the amplitude of the oscillation. The secular growth, on the other hand, is a purely numerical problem, probably related to the violation of the constraint equations. As for the oscillation amplitude, also the secular growth converges to zero with increasing resolution. Note that the convergence rate is not exactly second-order but slightly smaller, because the reconstruction schemes are only first-order accurate at local extrema (i.e. the centre and the surface of the star) thus increasing the overall truncation error [35].

In order to further investigate the accuracy of our implementation of the hydrodynamics equations, we have suppressed the spacetime evolution and solved just the hydrodynamics equations in the fixed spacetime of the initial TOV solution. This approximation is referred to as the "Cowling approximation" and is widely used in perturbative studies of oscillating stars. In this case, in addition to the confirmation of the convergence rate already checked in fully evolved runs, we have also compared the frequency spectrum of the numerically induced oscillation with the results obtained by an independent 2D code [84] and with perturbative analyses.

In Fig. 19 we show a comparison between the two codes reporting the power spectrum of the central density oscilla- 


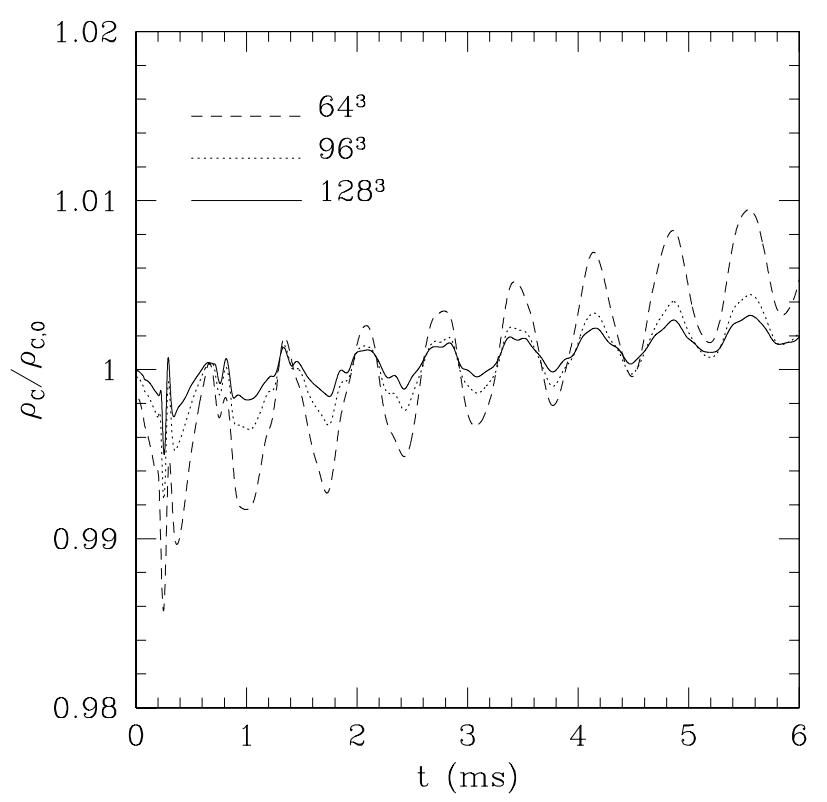

FIG. 18: Central mass-density, normalized to the initial value, in a stable TOV star $\left(M=1.4 M_{\odot}\right.$ and polytropic exponent $\left.\Gamma=2\right)$ evolution at different resolutions. PPM and Marquina were used for all runs.

tions computed with the Whisky code and the corresponding frequencies as obtained with perturbative techniques and with the $2 \mathrm{D}$ code. Clearly the agreement is very good with an error below $1 \%$ in the fundamental frequency. The fact that the frequencies computed with the code coincide with the physical eigenfrequencies calculated through perturbative analysis allows us to study with our code the physical properties of linear normal-modes of oscillation even if such oscillations are generated numerically.

The last test performed in the linear regime consists of the evolution of stationary solutions of rapidly rotating stars, with angular velocity up to $95 \%$ of the allowed mass-shedding limit for uniformly rotating stars. In this case, a number of small improvements on the boundary and gauge conditions have allowed us to extend considerably the timescale of our evolutions of stable rapidly rotating stars, which can now be evolved for about $10 \mathrm{~ms}$, a timescale which is 3 times larger than the one previously reported in [14]. In analogy with the non-rotating case, the truncation error triggers quasi-radial oscillations in the star. Such pulsations converge to zero with increasing resolution. Determining the frequency spectrum of fully relativistic and rapidly rotating stars is an important achievement, allowing the investigation of a parameter space which is astrophysically relevant but too difficult to treat with current perturbative techniques. More details on this investigation will be presented in a separate paper.

Finally, we have considered tests of the non-linear dynamics of isolated spherical relativistic stars. To this purpose we have constructed TOV solutions that are placed on the unstable branch of the equilibrium configurations (see inset of Fig. 20. The truncation error in the initial data for a TOV

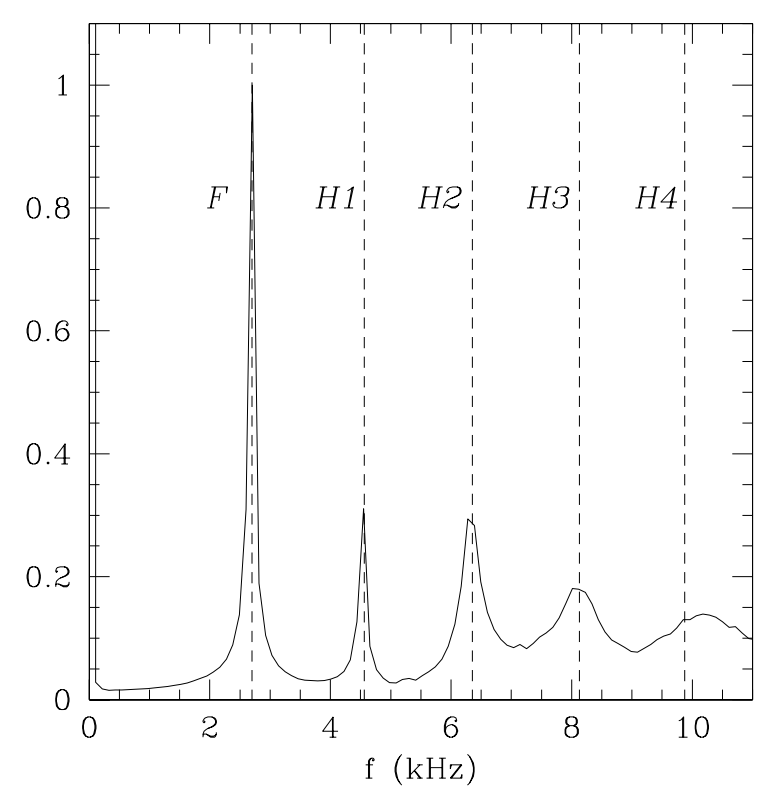

FIG. 19: Power spectrum of the central mass-density evolution of an $M=1.4 M_{\odot}, \Gamma=2$ stable TOV star performed with $128^{3}$ grid points. The units of the vertical axis are arbitrary.

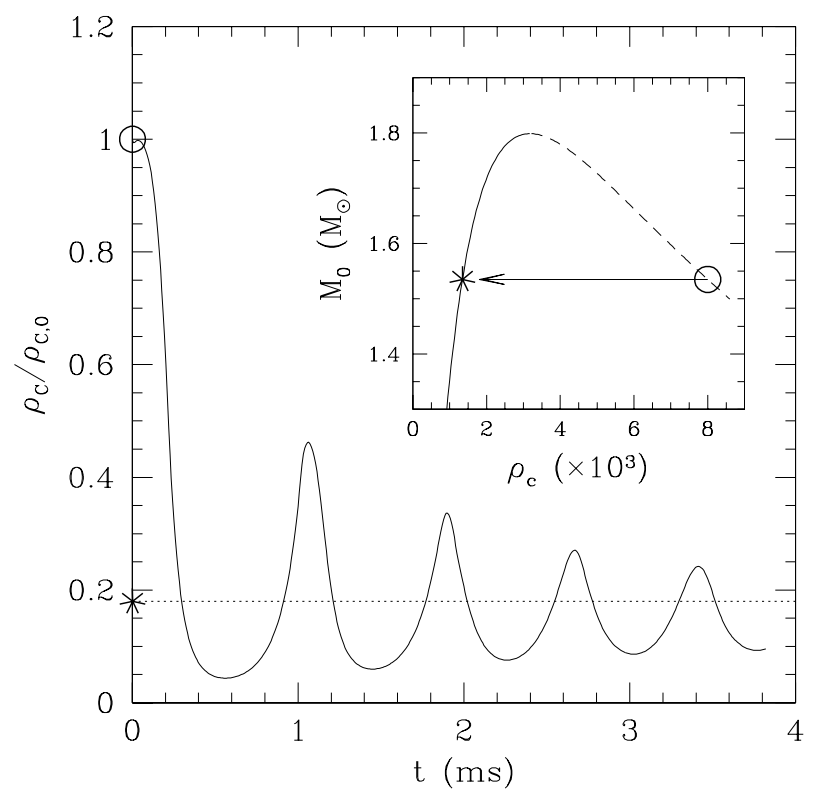

FIG. 20: Normalized central mass-density evolution of an $M=$ 1.4 $M_{\odot}, \Gamma=2$ unstable TOV star performed with $96^{3}$ grid points.

solution is sufficient to move the model to a different configuration and in Whisky this leads to a rapid migration toward a stable configuration of equal rest mass but smaller central density. Such a violent expansion produces large amplitude radial oscillations in the star that are either at constant amplitude, if the polytropic EOS 2.12 is used, or are damped through 
shock heating, if the ideal fluid EOS 2.14 is used and the equation for $\tau$ is evolved in time. A summary of this dynamics is presented in Fig. 20 which shows the time series of the normalized central density for a TOV solution. Note that the asymptotic central density tends to a value corresponding to a rest mass slightly smaller than the initial one (straight dotted line). This is the energy loss due to the internal dissipation.

\section{ACKNOWLEDGMENTS}

It is a pleasure to thank Joachim Frieben, José Mํㅡ. Ibáñez and Andrea Nerozzi who have participated to the development and testing of the code. We are also very grateful to Peter Di- ener, Jonathan Thornburg, Erik Schnetter, Badri Krishnan and Frank Herrmann for their help in the study of the trapped surfaces, to Abhay Ashtekar for clarifying some aspects of the dynamical-horizon framework, and to John Miller and Kōji Uryū for carefully reading the manuscript. We are also grateful to the Cactus-code team, for their efforts in producing an efficient infrastructure for numerical relativity. Financial support for this work has been provided by the MIUR and EU Network Programme (Research Training Network contract HPRN-CT-2000-00137). J.A.F. acknowledges financial support from the Spanish Ministerio de Ciencia y Tecnología (grant AYA 2001-3490-C02-01). The computations were performed on the Albert100 cluster at the University of Parma and the Peyote cluster at the Albert Einstein Institute.
[1] G. B. Cook, M. F. Huq, S. A. Klasky, M. A. Scheel, A. M. Abrahams, A. Anderson, P. Anninos, T. W. Baumgarte, N. Bishop, S. R. Brandt, et al., Phys. Rev. Lett 80, 2512 (1998).

[2] A. M. Abrahams, L. Rezzolla, M. E. Rupright, A. Anderson, P. Anninos, T. W. Baumgarte, N. T. Bishop, S. R. Brandt, J. C. Browne, K. Camarda, et al., Phys. Rev. Lett. 80, 1812 (1998).

[3] R. Gómez, L. Lehner, R. Marsa, J. Winicour, A. M. Abrahams, A. Anderson, P. Anninos, T. W. Baumgarte, N. T. Bishop, S. R. Brandt, et al., Phys. Rev. Lett. 80, 3915 (1998).

[4] M. Anderson and R. A. Matzner, submitted to Phys. Rev. D (2004), gr-qc/0307055.

[5] E. Schnetter, Ph.D. thesis, Universität Tübingen, Tübingen, Germany (2003), URN: urn:nbn:de:bsz:21-opus-8191, URL: http://w210.ub.uni-tuebingen.de/dbt/volltexte/2003/819/.

[6] S. Bonazzola, E. Gourgoulhon, P. Grandclément, and J. Novak, submitted to Phys. Rev. D (2004), gr-qc/0307082.

[7] L. Lehner, Class. Quantum Grav. 18, R25 (2001).

[8] T. Nakamura, K. Oohara, and Y. Kojima, Prog. Theor. Phys. Suppl. 90, 1 (1987).

[9] M. Shibata and T. Nakamura, Phys. Rev. D 52, 5428 (1995).

[10] T. W. Baumgarte and S. L. Shapiro, Physical Review D 59, 024007 (1999).

[11] M. Shibata, T. W. Baumgarte, and S. L. Shapiro, Phys. Rev. D 61, 044012 (2000).

[12] M. Shibata, Phys. Rev. D 67, 024033 (2003).

[13] M. Alcubierre, B. Brügmann, T. Dramlitsch, J. Font, P. Papadopoulos, E. Seidel, N. Stergioulas, and R. Takahashi, Phys. Rev. D 62, 044034 (2000).

[14] J. A. Font, T. Goodale, S. Iyer, M. Miller, L. Rezzolla, E. Seidel, N. Stergioulas, W. M. Suen, and M. Tobias, Phys. Rev. D 65, 084024 (2002).

[15] G. Yoneda and H. Shinkai, Phys. Rev. D 66, 124003 (2002).

[16] M. Kawamura, K. Oohara, and T. Nakamura, submitted to Prog. Theor. Phys. (2003), astro-ph/0306481.

[17] E. Seidel and W.-M. Suen, Phys. Rev. Lett. 69, 1845 (1992).

[18] S. Brandt, R. Correll, R. Gómez, M. F. Huq, P. Laguna, L. Lehner, P. Marronetti, R. A. Matzner, D. Neilsen, J. Pullin, et al., Phys. Rev. Lett. 85, 5496 (2000).

[19] M. Alcubierre and B. Brügmann, Phys. Rev. D 63, 104006 (2001).

[20] L. E. Kidder, M. A. Scheel, S. A. Teukolsky, E. D. Carlson, and G. B. Cook, Phys. Rev. D 62, 084032 (2000).

[21] L. E. Kidder, M. A. Scheel, and S. A. Teukolsky, Phys. Rev.
D 64, 064017 (2001).

[22] M. Alcubierre, B. Brügmann, D. Pollney, E. Seidel, and R. Takahashi, Phys. Rev. D 64, 61501 (R) (2001).

[23] H.-J. Yo, T. Baumgarte, and S. Shapiro, Phys. Rev. D 66, 084026 (2002).

[24] P. Laguna and D. Shoemaker, Class. Quantum Grav. 19, 3679 (2002).

[25] M. W. Choptuik, E. W. Hirschmann, S. L. Liebling, and F. Pretorius, Phys. Rev. D68, 044007 (2003).

[26] U. Sperhake, K. L. Smith, B. Kelly, P. Laguna, and D. Shoemaker (2003), gr-qc/0307015.

[27] M. M. May and R. H. White, in Methods in Computational Physics, edited by B. Alder, F. S., and R. M. (Academic Press, London, 1967), vol. 7, p. 129.

[28] M. D. Duez, S. L. Shapiro, and H.-J. Yo, Phys. Rev. D 69, 104016 (2004).

[29] T. Nakamura and M. Sasaki, Phys. Lett. 106 B, 69 (1981).

[30] T. Nakamura, Prog. Theor. Phys. 70, 1144 (1983).

[31] J. M. Bardeen and T. Piran, Phys. Reports 96, 205 (1983).

[32] R. F. Stark and T. Piran, Phys. Rev. Lett. 55, 891 (1985).

[33] R. F. Stark and T. Piran, in Proceedings of the Fourth Marcell Grossman Meeting on General Relativity, Rome, 1985, edited by R. Ruffini (Elsevier Science Publisher, 1986), p. 327.

[34] R. F. Stark and T. Piran, Comp. Phys. Rep. 5, 221 (1987).

[35] M. Alcubierre, W. Benger, B. Brügmann, G. Lanfermann, L. Nerger, E. Seidel, and R. Takahashi, Phys. Rev. Lett. 87, 271103 (2001).

[36] J. Frauendiener, Phys. Rev. D 66, 104027 (2002).

[37] M. Shibata, Prog. Theor. Phys. 104, 325 (2000).

[38] M. Shibata, Astrophys. J. 595, 992 (2003).

[39] L. Baiotti, I. Hawke, P. Montero, and L. Rezzolla, in Computational Astrophysics in Italy: Methods and Tools, edited by R. Capuzzo-Dolcetta (Mem. Soc. Astron. It. Suppl., Trieste, 2003), vol. 1, p. 327.

[40] J. A. Font, Living Rev. Relativity 6, 4 (2003), http://relativity.livingreviews.org/Articles/lrr-2003-4.

[41] European RTN on Sources of Gravitational Waves, www . eu-network . org.

[42] M. E. Rupright, A. M. Abrahams, and L. Rezzolla, Phys. Rev. D 58, 044005 (1998).

[43] L. Rezzolla, A. M. Abrahams, R. A. Matzner, M. Rupright, and S. Shapiro, Phys. Rev. D 59, 064001 (1999).

[44] E. Schnetter, S. H. Hawley, and I. Hawke, Class. Quantum Grav. 21, 1465 (2004). 
[45] L. Baiotti, I. Hawke, L. Rezzolla, and E. Schnetter, work in progress (2005).

[46] Cactus Computational Toolkit, www. cactuscode. org.

[47] J. A. Font, M. Miller, W. M. Suen, and M. Tobias, Phys. Rev. D 61, 044011 (2000).

[48] P. Collela and P. R. Woodward, J. Comput. Phys. 54, 174 (1984).

[49] A. Harten, B. Engquist, S. Osher, and S. R. Chakrabarty, J. Comput. Phys. 71, 2311 (1987).

[50] A. Harten, P. D. Lax, and B. van Leer, SIAM Rev. 25, 35 (1983).

[51] M. A. Aloy, J. M. Ibánez, J. M. Martí, and E. Müller, Astroph. J. Supp. 122, 151 (1999).

[52] J. Ibánez, M. Aloy, J. Font, J. Martí, J. Miralles, and J. Pons, in Godunov methods: theory and applications, edited by E. Toro (Kluwer Academic/Plenum Publishers, 2001).

[53] M. A. Aloy, J. A. Pons, and J. M. Ibánez, Comput. Phys. Commun. 120, 115 (1999).

[54] R. Arnowitt, S. Deser, and C. W. Misner, in Gravitation: An Introduction to Current Research, edited by L. Witten (John Wiley, New York, 1962), pp. 227-265.

[55] R. D. Richtmyer and K. Morton, Difference Methods for Initial Value Problems (Interscience Publishers, New York, 1967).

[56] J. W. York, Jr., in Sources of Gravitational Radiation, edited by L. L. Smarr (Cambridge University Press, Cambridge, UK, 1979), p. 83.

[57] M. Alcubierre, B. Brügmann, P. Diener, M. Koppitz, D. Pollney, E. Seidel, and R. Takahashi, Phys. Rev. D 67, 084023 (2003).

[58] O. Sarbach, G. Calabrese, J. Pullin, and M. Tiglio, Phys. Rev. D 66, 064002 (2002).

[59] C. Bona, T. Ledvinka, C. Palenzuela, and M. Zacek, Phys. Rev. D69, 064036 (2003).

[60] G. Nagy, O. E. Ortiz, and O. A. Reula (2004), gr-qc/0402123.

[61] M. Alcubierre, G. Allen, B. Brügmann, E. Seidel, and W. Suen, Phys. Rev. D 62, 124011 (2000).

[62] J. Balakrishna, G. Daues, E. Seidel, W.-M. Suen, M. Tobias, and E. Wang, Class. Quantum Grav. 13, L135 (1996).

[63] C. Bona, J. Massó, E. Seidel, and J. Stela, Phys. Rev. Lett. 75, 600 (1995).

[64] M. Alcubierre, Phys. Rev. D 55, 5981 (1997).

[65] M. Alcubierre and J. Massó, Phys. Rev. D 57, 4511 (1998).

[66] L. Smarr and J. York, Phys. Rev. D 17, 2529 (1978).

[67] J. M. Martí, J. M. Ibánez, and J. A. Miralles, Phys. Rev. D 43, 3794 (1991).

[68] F. Banyuls, J. A. Font, J. M. Ibánez, J. M. Martí, and J. A. Miralles, Astrophys. J. 476, 221 (1997).

[69] T. Zwerger, Ph.D. thesis, Technische Universität München, München, Germany (1995).

[70] T. Zwerger and E. Müller, Astron. Astrophys. 320, 209 (1997).

[71] P. D. Lax and B. Wendroff, Comm. Pure Appl. Math. 13, 217 (1960).

[72] T. Y. Hou and P. G. LeFloch, Math. of Comp. 62, 497 (1994).

[73] C. B. Laney, Computational Gasdynamics (Cambridge University Press, 1998).

[74] E. F. Toro, Riemann Solvers and Numerical Methods for Fluid Dynamics (Springer-Verlag, 1999).

[75] R. J. Leveque, in Computational Methods for Astrophysical Fluid Flow, edited by O. Steiner and A. Gautschy (SpringerVerlag, 1998).

[76] J. Thornburg, Classical and Quantum Gravity 21, 743 (2004).

[77] L. Kidder, M. Scheel, S. Teukolsky, and G. Cook, in Miniprogram on Colliding Black Holes: Mathematical Issues in $\mathrm{Nu}$ - merical Relativity (Institute for Theoretical Physics, UCSB, Santa Barbara, CA, 2000).

[78] G. Calabrese, L. Lehner, D. Neilsen, J. Pullin, O. Reula, O. Sarbach, and M. Tiglio, Class. Quant. Grav 20, L245 (2003).

[79] I. Hawke, F. Löffler, and A. Nerozzi (2005), gr-qc/0501054.

[80] J. L. Friedman, J. R. Ipser, and R. D. Sorkin, Astrophys. J. 325, 722 (1988).

[81] N. Stergioulas and J. L. Friedman, Astrophys. J. 444, 306 (1995).

[82] N. Stergioulas and J. Font, Phys. Rev. Lett. 86, 1148 (2001).

[83] N. Stergioulas, Living Rev. Relativity 6, 3 (2003).

[84] J. A. Font, N. Stergioulas, and K. D. Kokkotas, Mon. Not. R. Astron. Soc. 313, 678 (2000).

[85] N. Stergioulas and I. Hawke, in Recent Developments in Gravity, Proceedings of the 10th Hellenic Relativity Conference, edited by K. D. Kokkotas and N. Stergioulas (World Scientific, Singapore, 2003), p. 185.

[86] G. B. Cook, Living Rev. Relativity 5, 5 (2000).

[87] S. E. Woosley, in Proceedings of the International Workshop held in Rome, CNR headquarters, October, 2000, edited by E. Costa, F. Frontera, , and J. Hjorth (Springer, Berlin Heidelberg, 2000), p. 257.

[88] O. Zanotti, L. Rezzolla, and J. A. Font, MNRAS 341, 832 (2003).

[89] M. Shibata, Phys. Rev. D 60, 104052 (1999).

[90] H. Dimmelmeier, J. A. Font, and E. Müller, Astron. and Astrophys. 393, 523 (2002).

[91] M. Shibata and K. Uryu, Prog. Theor. Phys. 107, 265 (2002).

[92] S. L. Shapiro, Astrophys. J. 544, 397 (2000).

[93] J. N. Cook, S. L. Shapiro, and B. C. Stephens, Astrophys. J. 599, 1272 (2003).

[94] Y. T. Liu and S. L. Shapiro, Phys. Rev. D 69, 044009 (2004).

[95] J. Bardeen, Astrophys. J. 162, 171 (1970).

[96] A. Cumming, S. M. Morsink, L. Bildsten, J. L. Friedman, and D. E. Holz, Astrophys. J. 564, 343 (2000).

[97] L. Rezzolla, F. K. Lamb, and S. L. Shapiro, Astrophys. J. 531, L139 (2000).

[98] H. C. Spruit, Astron. and Astrophys. 341, L1 (1999).

[99] P. Diener, Class. Quantum Grav. 20, 4901 (2003).

[100] P. Anninos, D. Bernstein, S. R. Brandt, D. Hobill, E. Seidel, and L. Smarr, Phys. Rev. D 50, 3801 (1994).

[101] P. Anninos, D. Bernstein, S. Brandt, J. Libson, J. Massó, E. Seidel, L. Smarr, W.-M. Suen, and P. Walker, Phys. Rev. Lett. 74, 630 (1995).

[102] P. Anninos, D. Bernstein, S. Brandt, D. Hobill, E. Seidel, and L. Smarr, Austral. Journ. Phys. 48, 1027 (1995).

[103] S. Brandt and E. Seidel, Phys. Rev. D 52, 870 (1995).

[104] S. Brandt, J. A. Font, J. M. Ibáñez, J. Massó, and E. Seidel, Comp. Phys. Comm. 124, 169 (2000).

[105] S. Brandt and E. Seidel, Phys. Rev. D 52, 856 (1995).

[106] A. Ashtekar, C. Beetle, and S. Fairhurst, Class. Quantum Grav. 17, 253 (2000).

[107] A. Ashtekar, C. Beetle, O. Dreyer, S. Fairhurst, B. Krishnan, J. Lewandowski, and J. Wisniewski, Phys. Rev. Lett. 85, 3564 (2000).

[108] A. Ashtekar, C. Beetle, and J. Lewandowski, Phys. Rev. D 64, 044016 (2001).

[109] A. Ashtekar and B. Krishnan, Phys. Rev. Lett. 89, 261101 (2002).

[110] O. Dreyer, B. Krishnan, D. Shoemaker, and E. Schnetter, Phys. Rev. D 67, 024018 (2002).

[111] A. Ashtekar and B. Krishnan, Phys. Rev. D 68, 104030 (2003).

[112] D. Christodoulou, Phys. Rev. Lett. 25, 1596 (1970). 
[113] C. W. Shu and S. J. Osher, Journ. Comput. Phys. 77, 439 (1988).

[114] S. Gottlieb and C. Shu, Math. Comp. 67, 73 (1998).

[115] C. W. Shu, in High-Order Methods for Computational Physics, edited by T. J. Barth and H. Deconinck (Springer, 1999).

[116] J. A. Pons, J. M. Martí, and E. Müller, Journ. Fluid Mech. 422, 125 (2000).

[117] L. Rezzolla and O. Zanotti, Journ. Fluid. Mech. 449, 395 (2001).
[118] L. Rezzolla, O. Zanotti, and J. A. Pons, Journ. Fluid. Mech. 479, 199 (2003).

[119] P. L. Roe, J. Comput. Phy. 43, 357 (1981).

[120] R. Donat and A. Marquina, Journ. Comput. Phys. 125, 42 (1996).

[121] R. Donat, J. A. Font, J. M. Ibánez, and A. Marquina, Journ. Comput. Phys. 146, 58 (1998).

[122] M. D. Duez, P. Marronetti, S. L. Shapiro, and T. W. Baumgarte, Phys. Rev. D67, 024004 (2003). 